\title{
Organic Haze as a Biosignature in Anoxic Earth-like Atmospheres
}

\author{
Giada Arney,, ${ }^{1,2}$ Shawn D. Domagal-Goldman,, ${ }^{1,2}$ and Victoria S. Meadows ${ }^{2,3,4}$
}

\begin{abstract}
Early Earth may have hosted a biologically mediated global organic haze during the Archean eon (3.8-2.5 billion years ago). This haze would have significantly impacted multiple aspects of our planet, including its potential for habitability and its spectral appearance. Here, we model worlds with Archean-like levels of carbon dioxide orbiting the ancient Sun and an M4V dwarf (GJ 876) and show that organic haze formation requires methane fluxes consistent with estimated Earth-like biological production rates. On planets with high fluxes of biogenic organic sulfur gases $\left(\mathrm{CS}_{2}, \mathrm{OCS}, \mathrm{CH}_{3} \mathrm{SH}\right.$, and $\left.\mathrm{CH}_{3} \mathrm{SCH}_{3}\right)$, photochemistry involving these gases can drive haze formation at lower $\mathrm{CH}_{4} / \mathrm{CO}_{2}$ ratios than methane photochemistry alone. For a planet orbiting the Sun, at $30 \times$ the modern organic sulfur gas flux, haze forms at a $\mathrm{CH}_{4} / \mathrm{CO}_{2}$ ratio $20 \%$ lower than at $1 \times$ the modern organic sulfur flux. For a planet orbiting the M4V star, the impact of organic sulfur gases is more pronounced: at $1 \times$ the modern Earth organic sulfur flux, a substantial haze forms at $\mathrm{CH}_{4} / \mathrm{CO}_{2} \sim 0.2$, but at $30 \times$ the organic sulfur flux, the $\mathrm{CH}_{4} / \mathrm{CO}_{2}$ ratio needed to form haze decreases by a full order of magnitude. Detection of haze at an anomalously low $\mathrm{CH}_{4} /$ $\mathrm{CO}_{2}$ ratio could suggest the influence of these biogenic sulfur gases and therefore imply biological activity on an exoplanet. When these organic sulfur gases are not readily detectable in the spectrum of an Earth-like exoplanet, the thick organic haze they can help produce creates a very strong absorption feature at UV-blue wavelengths detectable in reflected light at a spectral resolution as low as 10. In direct imaging, constraining $\mathrm{CH}_{4}$ and $\mathrm{CO}_{2}$ concentrations will require higher spectral resolution, and $R>170$ is needed to accurately resolve the structure of the $\mathrm{CO}_{2}$ feature at $1.57 \mu \mathrm{m}$, likely the most accessible $\mathrm{CO}_{2}$ feature on an Archean-like exoplanet. Key Words: Organic haze-Organic sulfur gases-Biosignatures-Archean Earth. Astrobiology 18, 311-329.
\end{abstract}

\section{Introduction}

T HE ArCheAn EON (3.8-2.5 billion years ago) may have experienced several intervals when a transient organic haze globally veiled our planet (e.g. Trainer et al., 2006; Zerkle et al., 2012; Izon et al., 2015, 2017; Hicks et al., 2016). This haze would have dramatically altered our planet's climate, spectral appearance, and photochemistry (Pavlov et al., 2001a, 2001b; Domagal-Goldman et al., 2008; Haqq-Misra et al., 2008; Wolf and Toon, 2010; Hasenkopf et al., 2011; Kurzweil et al., 2013; Claire et al., 2014; Arney et al., 2016, 2017). Organic haze formation is driven by methane photochemistry, and its optical thickness is controlled by the ratio of the amount of atmospheric methane $\left(\mathrm{CH}_{4}\right)$ relative to the amount of carbon dioxide $\left(\mathrm{CO}_{2}\right)$ (e.g.,
Trainer et al., 2006) because oxygen radicals produced by $\mathrm{CO}_{2}$ photolysis can frustrate organic haze formation in Earthlike atmospheres (Arney et al., 2017).

On Archean Earth, there are numerous potential sources of methane, although biological processes likely dominated, as they do today (Kharecha et al., 2005). Methanogenesis, an anaerobic metabolism deeply rooted in the tree of life that likely evolved early in Earth's history (Woese and Fox, 1977; Ueno et al., 2006), involves the uptake of $\mathrm{CO}_{2}$ and $\mathrm{H}_{2}$ to form $\mathrm{CH}_{4}$ and $\mathrm{H}_{2} \mathrm{O}$ :

$$
\mathrm{CO}_{2}+4 \mathrm{H}_{2} \rightarrow \mathrm{CH}_{4}+2 \mathrm{H}_{2} \mathrm{O}
$$

Methanogenesis has only been observed in types of archaea, and it occurs in a number of anoxic environments on

\footnotetext{
${ }^{1}$ NASA Goddard Space Flight Center, Greenbelt, Maryland.

${ }^{2}$ NASA Astrobiology Institute Virtual Planetary Laboratory, University of Washington, Seattle, Washington.

${ }^{3}$ Astronomy Department, University of Washington, Seattle, Washington

${ }^{4}$ University of Washington Astrobiology Program, Seattle, Washington.

(c) Giada Arney et al., 2018; Published by Mary Ann Liebert, Inc. This Open Access article is distributed under the terms of the Creative Commons Attribution Noncommercial License (http://creativecommons.org/licenses/by-nc/4.0/) which permits any noncommercial use, distribution, and reproduction in any medium, provided the original author(s) and the source are credited.
} 
modern Earth including animal guts and hydrothermal vents (Ver Eecke et al., 2012). In the latter environment, methanogens react $\mathrm{H}_{2}$ contained in reduced vent fluids with $\mathrm{CO}_{2}$ dissolved in the seawater. On Earth today, the flux of methane produced by biology is roughly $10^{11}$ molecules/ $\mathrm{cm}^{2} / \mathrm{s}$ (Pavlov et al., 2001a), and the Archean biotic flux has been estimated to range somewhere between $1 / 3$ and 2.5 times this modern value (Kharecha et al., 2005).

The abiotic production rate of methane is not as well constrained. Serpentinization, the hydration of olivine and pyroxene, is the dominant abiotic source of methane on Earth today (Kelley et al., 2005; Etiope and Sherwood Lollar, 2013; Guzmán-Marmolejo et al., 2013). The source of methane from serpentinizing systems is not completely clear. Serpentinization produces $\mathrm{H}_{2}$, and it has been suggested that this $\mathrm{H}_{2}$ can then react with $\mathrm{CO}_{2}$ or $\mathrm{CO}$ to form $\mathrm{CH}_{4}$ through Fischer-Tropsch-type reactions (Bradley and Summons, 2010). However, other explanations for methane produced in serpentinizing systems have been offered. For example, methanogens are known to live in these systems, so some fraction of the methane produced from the vents is biological (Brazelton et al., 2006). However, based on the total density of cells expected in the vent systems, it is unlikely that methanogens are the dominant methane source in at least the Lost City hydrothermal system (Bradley and Summons, 2010). More recently, the isotopic composition of the hydrocarbons emanating from the Von Damm hydrothermal system was analyzed in relation to the isotopic composition of dissolved inorganic carbon (McDermott et al., 2015). Surprisingly, the isotopic composition of the vented $\mathrm{CH}_{4}$ indicates that little to none of the $\mathrm{CH}_{4}$ coming out the Van Damm vents is produced by reduction of dissolved inorganic carbon circulating through the rocks and participating in Fischer-Tropsch-type chemistry. Possibly, the $\mathrm{CH}_{4}$ produced in these systems is released from trapped magmatic fluid inclusions. Consistent with this finding, McCollom (2016) conducted laboratory experiments on the serpentinization of olivine with and without pyroxene using ${ }^{13} \mathrm{C}$-labeled $\mathrm{CO}_{2}$ and found that production rates of ${ }^{13} \mathrm{CH}_{4}$ were inefficient. ${ }^{13} \mathrm{C}$-labeled $\mathrm{CH}_{4}$ was produced in only one of their experiments: this experiment also contained a dissolved $\mathrm{H}_{2}$-rich vapor phase that may help promote FischerTropsch-type reactions. The kinetic barriers to methane formation in these reactions are surmountable in the presence of iron-nickel-phase mineral catalysts such as awaurite, although the McDermott et al. (2015) study of the Von Damm vent implies that at least in some serpentinizing systems these reactions are not important for the bulk methane production and another source dominates.

Kasting and Catling (2003) estimated the abiotic flux of methane as $1 / 300^{\text {th }}$ the biotic rate. However, more recent measurements by Kelley et al. (2005) at the Lost City hydrothermal system indicate that abiotic methane production rates may actually be as high as $1 / 30^{\text {th }}$ the biotic flux.

A different line of argument to estimate the abiotic methane production from serpentinization on Earth comes from Guzmán-Marmolejo et al. (2013). These authors calculate how much methane can be produced given current crustal spreading rates as a function of available $\mathrm{FeO}$ in the crust and by considering $\mathrm{CO}_{2}$ as the limiting reactant for methane production in serpentinization-however, note that if the $\mathrm{CH}_{4}$ is instead produced by a different mechanism, this assumption may no longer apply. Based on the assumption of Fischer-Tropsch-type synthesis, they estimate that the maximum amount of abiotic methane that can be produced from serpentinization is $6.8 \times 10^{8}$ molecules $/ \mathrm{cm}^{2} / \mathrm{s}$ for a 1-Earth-mass planet, which is only $1 / 160^{\text {th }}$ the biotic flux (Guzmán-Marmolejo et al. [2013] also estimate the maximum $\mathrm{CH}_{4}$ production rate as $1.3 \times 10^{9}$ molecules $/ \mathrm{cm}^{2} / \mathrm{s}$ for a 5-Earth-mass planet). However, note that these results are contingent on the assumption of the modern-day Earth crustal spreading rate and the source of the methane.

Considering all the above estimates, abiotic methane production estimates from serpentinization ranging between approximately $1 / 30^{\text {th }}$ and $1 / 150^{\text {th }}$ the present biotic flux appear reasonable for modern Earth. In the Archean, abiotic methane production rates are even less certain than they are today. If the early planet had faster seafloor spreading rates or a higher fraction of seafloor ultramafic rocks, enhanced abiotic methane compared to the modern planet would have been possible (Kasting, 2005; Shaw, 2008). Faster seafloor spreading rates may be more likely on a hotter young planet where convection may have proceeded more efficiently. How tectonics operated in the Archean remains uncertain, but there is geological and numerical modeling evidence supporting the existence of plate tectonics during this period (Kerrich and Polat, 2006).

Although serpentinization is thought to be the dominant source of abiotic methane on Earth today, there are other abiotic methane sources (Etiope and Sherwood Lollar, 2013). These sources include primordial delivery (via exogeneous sources); internal magmatic, postmagmatic, and metamorphic processes; iron-carbonate decomposition; carbonate methanation; and aqueous $\mathrm{CO}_{2}$ reduction. Global emissions of all non-anthropogenic biotic methane fluxes to the atmosphere have been well studied and constrained at approximately $200 \mathrm{Mt} /$ year (Core Writing Team et al., 2007). Total estimated methane emissions from geological sources amount to about $60 \mathrm{Mt} /$ year (about $1 \times 10^{10}$ molecules $/ \mathrm{cm}^{2} / \mathrm{s}$ ), although this flux is probably not purely abiotic (Etiope, 2012): Etiope and Sherwood Lollar (2013) discuss how volcanic and nonvolcanic geothermal systems can also release methane derived from thermal breakdown of sedimentary rock organic matter, which can be biotic (Etiope et al., 2007). The total global emission of truly abiotic methane is not well constrained. Emmanuel and Ague (2007) suggest it may be as low as $\sim 2.3 \mathrm{Mt} / \mathrm{year}$ (about $4 \times 10^{8}$ molecules $/ \mathrm{cm}^{2} / \mathrm{s}$ ), although Etiope and Sherwood Lollar (2013) point out that this estimate is hypothetical and not has not been constrained by direct measurements. Of course, we emphasize again that all these estimates are for modern-day Earth; past Earth, and other planets, will naturally have different methane flux rates.

Given all of this, finding an organic haze in the atmosphere of a planet with Archean-like $\mathrm{CO}_{2}$ levels could be indicative of highly interesting processes that imply ongoing geological activity and/or biological methane production with high methane source fluxes to drive haze production in a $\mathrm{CO}_{2}$-rich atmosphere. Such a planet should be a target of closer follow-up studies aimed at discriminating between geological and biological $\mathrm{CH}_{4}$ sources and to search for other signs of habitability and life (Section 4.3). We emphasize that while detection of $\mathrm{CH}_{4}$ and organic hazewhich can have a significantly stronger spectral signature 
than $\mathrm{CH}_{4}$-in an Earth-like atmosphere would be a tantalizing hint of the presence of methane-producing life on an exoplanet (since biology produces the bulk of methane on modern Earth), it would not be enough to conclude biological activity given the existence of abiotic $\mathrm{CH}_{4}$ sources. This is similar to-but perhaps more obvious than- the case for oxygen as a biosignature given that several pathways for abiotic oxygen production have recently come to light (Domagal-Goldman et al., 2014; Harman et al., 2015; Luger and Barnes, 2015; Schwieterman et al., 2016). The spectral signatures of possibly biologically produced spectral features need to be placed in the context of a broader understanding of that planet's atmospheric chemistry and potential for habitability. In this manuscript, we pursue one type of contextual information that would help to discriminate between abiotic and biotic hazes on Earth-like worlds.

It has been pointed out that biogenic organic sulfur gases $\left(\mathrm{S}_{\text {org }}\right)$ can contribute to the atmospheric hydrocarbon budget through photochemical processes that liberate organic species from the $\mathrm{S}_{\text {org }}$ molecules (Domagal-Goldman et al., 2011). These $S_{\text {org }}$ gases include carbon disulfide $\left(\mathrm{CS}_{2}\right)$, carbonyl sulfide (OCS), methanethiol $\left(\mathrm{CH}_{3} \mathrm{SH}\right.$, also called methyl mercaptan), and dimethyl sulfide $\left(\mathrm{CH}_{3} \mathrm{SCH}_{3}\right.$ or DMS). Volcanic processes can also produce $\mathrm{CS}_{2}$ and OCS, but at lower fluxes than Earth's biology (Lomans et al., 2002). The potential for $S_{\text {org }}$ gases to act as biosignatures has been considered by previous studies (Pilcher, 2003; Vance et al., 2011), and Domagal-Goldman et al. (2011) showed that although $\mathrm{S}_{\text {org }}$ gases may be difficult to directly detect in a planet's spectrum, their photochemical byproducts can produce spectral signatures that can indirectly imply a flux of these gases. In particular, Domagal-Goldman et al. (2011) found the photolysis of $\mathrm{S}_{\text {org }}$ gases can release methyl radicals $\left(\mathrm{CH}_{3}\right)$ that contribute to ethane $\left(\mathrm{C}_{2} \mathrm{H}_{6}\right)$ production in excess of the amount predicted from methane photochemistry alone. This effect is especially pronounced around $\mathrm{M}$ dwarfs whose UV spectral output allows for longer atmospheric lifetimes of $\mathrm{C}_{2} \mathrm{H}_{6}$ than solar-type stars. Unfortunately, $\mathrm{C}_{2} \mathrm{H}_{6}$ absorbs most strongly in the mid-IR at $12 \mu \mathrm{m}$, making its detection potentially difficult. DomagalGoldman et al. (2011) did not consider $\mathrm{S}_{\text {org }}$-rich atmospheres with enough methane to lead to haze formation, nor did they simulate the spectral region in which hazes have detectable features. But the same principles that caused higher $\mathrm{C}_{2} \mathrm{H}_{6}$ in their simulations could also cause a greater haze concentration in the presence of $S_{\text {org }}$. Here, we will test whether the hydrocarbons contributed to the atmosphere by $\mathrm{S}_{\text {org }}$ photochemistry can induce haze formation at lower $\mathrm{CH}_{4} / \mathrm{CO}_{2}$ ratios than would be expected if haze formation was driven by methane production alone, thereby providing a spectral clue that biological activities may be influencing haze formation on a planet. Organic haze is a particularly useful potential biosignature because it produces a very strong broadband absorption feature at UV and visible wavelengths (this is the reason why Titan is orange), and it also produces absorption features in the near infrared (NIR). These features may be accessible with observatories becoming available in the coming decades, including the James Webb Space Telescope (JWST) and possible future large direct-imaging telescopes such as the Large UV Optical Infrared telescope (LUVOIR) and the Habitable Exoplanet Imaging Mission (HabEx) (Postman et al., 2010;
Bolcar et al., 2015; Dalcanton et al., 2015; Mennesson et al., 2016), as we have studied previously (Arney et al., 2017).

\section{Methods}

To simulate Archean-analog planets, we use a coupled 1D photochemical-climate model called Atmos. The Atmos model is described in detail in Arney et al. (2016), and limitations of the Atmos haze formation scheme are discussed in Arney et al. (2016) and Arney et al. (2017). In brief, this model assumes a pathway proposed for Titan's hazes (Allen et al., 1980; Yung et al., 1984) where haze formation occurs via polymerization of acetylene $\left(\mathrm{C}_{2} \mathrm{H}_{2}\right)$. In this scheme, haze particles are formed via $\mathrm{C}_{2} \mathrm{H}+\mathrm{C}_{2} \mathrm{H}_{2} \rightarrow$ $\mathrm{C}_{4} \mathrm{H}_{2}$ and $\mathrm{C}_{2} \mathrm{H}+\mathrm{CH}_{2} \mathrm{CCH}_{2} \rightarrow \mathrm{C}_{5} \mathrm{H}_{4}+\mathrm{H}$. However, Cassini measurements of Titan show that haze formation is more complex and includes ion chemistry and the formation of nitriles (Waite et al., 2007; Vuitton et al., 2009; LópezPuertas et al., 2013). Additionally, while Titan's atmosphere is extremely reducing, Archean Earth's atmosphere would have been less so, and laboratory studies have shown that oxygen atoms can be incorporated into haze molecules (Trainer et al., 2006; DeWitt et al., 2009; Hörst and Tolbert, 2014; Hicks et al., 2016). Lack of these processes in Atmos may cause the model to underpredict the haze formation rate; on the other hand, in a real atmosphere, $\mathrm{C}_{4} \mathrm{H}_{2}$ would be able to revert back to $\mathrm{C}_{2} \mathrm{H}_{2}$. Our model does not include this, which could lead to haze overprediction. Ongoing improvements to Atmos will include a more complete haze formation scheme.

The climate portion of Atmos was originally developed by Kasting and Ackerman (1986), although it has been significantly modernized since then, and it was most recently updated and described in a recalculation of habitable zone boundaries around main sequence stars (Kopparapu et al., 2013) and in a study of the impact of organic haze in the Archean (Arney et al., 2016). The photochemical portion of Atmos is based on a code developed by Kasting et al. (1979), and it was significantly modernized by Zahnle et al. (2006). This model, supported by the Virtual Planetary Laboratory in NASA's Astrobiology Institute, is now publicly available at https://github.com/VirtualPlanetaryLaboratory/atmos.

The climate model is considered converged when the change in flux out the top of the atmosphere and change in surface temperature are sufficiently small (typically on the order of $1 \times 10^{-5}$ ) and when the energy from the star into the atmosphere balances the energy radiated out of the atmosphere. The photochemical model uses a first-order reverse Euler solver to solve continuity and flux equations for each species at all altitudes. In the time-stepping loop, the model tracks how much the gas concentrations change in each step, and the species with the largest relative error in its change in concentration (called $E_{\max }$ ) is used to set the size of the next time step. When $E_{\max }$ is small, the next time step will be larger; if $E_{\max }$ is too large, the model will decrease the timestep size. The model checks the time-step length to determine convergence, and when the time-step size exceeds $1 \times 10^{17} \mathrm{~s}$, the model considers itself "converged" and stops.

Both the climate and photochemical models have been modified to simulate haze particles as fractal in shape rather than as spherical (Mie) particles (Wolf and Toon, 2010; Zerkle et al., 2012; Arney et al., 2016) by using the fractal 
mean field approximation (Botet et al., 1997). Studies of Titan's atmosphere indicate that fractal particles are more realistic for organic hazes (Rannou et al., 1997), and early Earth-analog fractal organic hazes have been simulated in the laboratory (Trainer et al., 2006). Zerkle et al. (2012) and Arney et al. (2016) describe our model's haze particle treatment in detail. Fractal particles are composed of multiple smaller spherical particles called monomers clumped together into complex branching forms, and their scattering and absorption physics differ from spherical particles. In general, compared to equal-mass spherical particles, fractal particles produce more extinction at shorter wavelengths and less extinction at longer wavelengths. The consequences of this behavior have been previously considered in the context of UV-shielding and climate cooling effects for an Archean haze (Wolf and Toon, 2010; Zerkle et al., 2012; Arney et al., 2016). Generally, haze particles initially form at altitudes of $80-90 \mathrm{~km}$; this altitude is a result of the model's photochemistry and is not prescribed.

The methyl radicals produced by $\mathrm{S}_{\text {org }}$ gases that participate in haze formation photochemistry can be generated by reactions such as

$$
\begin{gathered}
\mathrm{CH}_{3} \mathrm{SH}+\mathrm{O} \rightarrow \mathrm{CH}_{3}+\mathrm{HSO} \\
\mathrm{CH}_{3} \mathrm{SH}+h \nu \rightarrow \mathrm{CH}_{3}+\mathrm{HS}
\end{gathered}
$$

The full chemical network that $S_{\text {org }}$ gases participate in is discussed in detail in Domagal-Goldman et al. (2011). Once $\mathrm{CH}_{3}$ is produced, it directly contributes to haze formation via the process outlined in Arney et al. (2017): $\mathrm{CH}_{3}$ produces ethane most efficiently through $\mathrm{CH}_{3}+\mathrm{CH}_{3} \mathrm{CO} \rightarrow$ $\mathrm{C}_{2} \mathrm{H}_{6}+\mathrm{CO}$. Ethane can then be photolyzed to produce $\mathrm{C}_{2} \mathrm{H}_{4}$ or $\mathrm{C}_{2} \mathrm{H}_{2}$, or it can react with $\mathrm{OH}$ to produce $\mathrm{C}_{2} \mathrm{H}_{5}$, all of which step toward haze formation.

Spectra are generated by the Spectral Mapping Atmospheric Radiative Transfer model (SMART) (Meadows and Crisp, 1996; Crisp, 1997) using outputs from the Atmos model. SMART is a 1D, line-by-line, fully multiple scattering radiative transfer model. Haze is included in SMART from Atmos via a particle binning scheme described in Arney et al. (2016). The newest version of SMART can also calculate transit transmission spectra in the same model run that calculates reflected light spectra. The model's transit calculations include the path length and refraction effects inherent in transit transmission spectra (Misra et al., 2014a, 2014b).

To simulate observations with possible large future spacebased telescopes, we use the coronagraph noise model described in Robinson et al. (2016). The same nominal parameters are assumed as those discussed in Robinson et al., except we assume a telescope operating temperature of $270 \mathrm{~K}$ and a constant quantum efficiency as a function of wavelength (0.9). Noise sources include dark noise, read noise, zodiacal and exozodiacal light, stellar light leakage, telescope thermal radiation. A publically accessible online version of this simulator is available at https://asd.gsfc.nasa .gov/luvoir/tools.

\subsection{Model inputs}

We compare haze production under the influence of $S_{\text {org }}$ gases for planets orbiting the Sun 2.7 billion years ago $(\mathrm{Ga})$ during the Archean eon and the M4V dwarf GJ 876 with the same total insolation as the $2.7 \mathrm{Ga}$ Sun $\left(0.8 \times 1360 \mathrm{~W} / \mathrm{m}^{2}\right)$. GJ 876 is a known multiplanet host (Von Braun et al., 2014). The spectrum we use for it is described in DomagalGoldman et al. (2014) based on the spectrum reported in France et al. (2012). We chose this star over higher-activity M dwarfs (e.g., AD Leo) because Domagal-Goldman et al. (2011) showed that $S_{\text {org }}$ gases have a greater impact on hydrocarbon photochemistry for lower-activity $\mathbf{M}$ dwarfs. Stars with lower UV outputs generate fewer photochemical oxygen species, which are major sinks of both hydrocarbons and organic sulfur gases (Domagal-Goldman et al., 2011; Arney et al., 2017). For the Sun, we corrected its spectrum for higher levels of expected activity when it was younger using the wavelength-dependent solar evolution correction from Claire et al. (2012), which estimates the solar flux at different epochs by combining data from the Sun and solar analogues to determine appropriate wavelength-dependent stellar flux corrections. The stellar spectra used in this study are shown for the UV, visible, and NIR in Fig. 1. The UV spectra shown in Fig. 1 show the actual resolution of the wavelength grid used by the photochemical model. The model's "Lyman alpha" bin encompasses flux from wavelengths spanning $8 \AA$ wide on either side of Lyman alpha (121.6 nm).

Our chemical reaction network is based on the one used by Arney et al. (2016) and Arney et al. (2017), although these earlier studies did not include $\mathrm{S}_{\text {org }}$ gases. The supplementary online information of Arney et al. (2016) provides a complete list of chemical reactions and species boundary conditions for this nominal Archean model. To
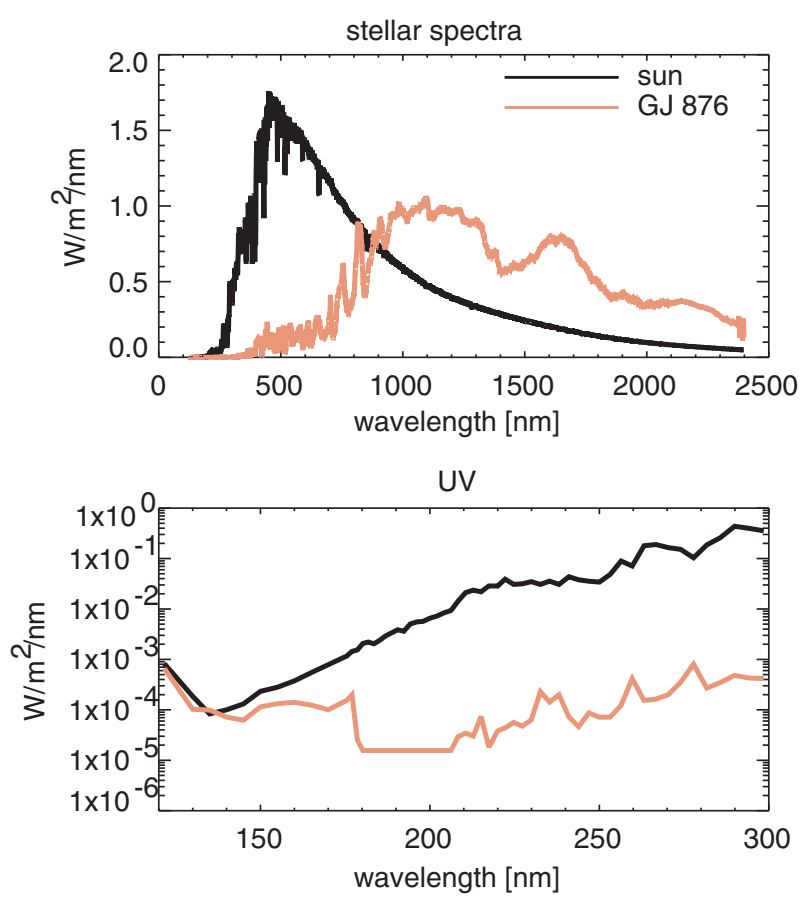

FIG. 1. Spectra of the Archean Sun and GJ 876 used in this study. The bottom panel shows the actual UV wavelength grid used in the photochemical model. Wavelength bins are larger than individual emission lines (e.g., Lyman alpha). 
include $\mathrm{S}_{\text {org }}$, we updated our templates based on the reaction list and boundary conditions discussed in DomagalGoldman et al. (2011). However, we removed the $\mathrm{NH}_{3^{-}}$ related gases and reactions from the templates shown in Domagal-Goldman et al. (2011) because we have not yet incorporated $\mathrm{NH}_{3}$ into our climate model, and it is a potentially significant greenhouse gas. Inclusion of $\mathrm{NH}_{3}$ remains an important area of future work because it may play a role in warming our early planet under the fainter young Sun, especially under a haze that could protect it from UV photolysis (Sagan and Chyba, 1997; Pavlov et al., 2001a; Wolf and Toon, 2010). Our atmospheres with $1 \times S_{\text {org }}$ fluxes use the same $S_{\text {org }}$ surface boundary fluxes presented in Table 2 of Domagal-Goldman et al. (2011). In units of molecules $/ \mathrm{cm}^{2} / \mathrm{s}$, these fluxes are $1.4 \times 10^{7}$ for $\mathrm{CS}_{2}$ and OCS, $8.3 \times 10^{8}$ for $\mathrm{CH}_{3} \mathrm{SH}, 4.2 \times 10^{9}$ for $\mathrm{CH}_{3} \mathrm{SCH}_{3}$, and 0 for $\mathrm{CH}_{3} \mathrm{~S}_{2} \mathrm{CH}_{3}$. This last species, $\mathrm{CH}_{3} \mathrm{~S}_{2} \mathrm{CH}_{3}$, or dimethyl disulfide (DMDS), is not produced by biology but results from $S_{\text {org }}$ photochemistry. Other nonbiological $S_{\text {org }}$ gases relevant to $S_{\text {org }}$ photochemistry included in our photochemical scheme are CS $\left(1.7 \times 10^{7}\right.$ molecules $/ \mathrm{cm}^{2} / \mathrm{s}$ produced at the surface $)$, and $\mathrm{CH}_{3} \mathrm{~S}\left(0\right.$ molecules $/ \mathrm{cm}^{2} / \mathrm{s}$ produced at the surface, but photochemistry can produce this gas in the atmosphere).

Unlike our previous studies where we set the $\mathrm{CH}_{4}$ surface mixing ratio to explore haze formation under different $\mathrm{CH}_{4}$ concentrations (Arney et al., 2016, 2017), here we vary the $\mathrm{CH}_{4}$ surface flux and allow the photochemical model to calculate the self-consistent atmospheric mixing ratio from the selected fluxes to explore haze formation under different $\mathrm{CH}_{4}$ production rates. The $\mathrm{CO}_{2}$ atmospheric fractions $\left(f \mathrm{CO}_{2}\right)$ simulated here range from $1 \times 10^{-5}$ to $1 \times 10^{-1}$; the lower limit on $\mathrm{CO}_{2}$ abundance was chosen to roughly represent the limit for $\mathrm{C} 4$ photosynthesis (Kestler et al., 1975; Tolbert et al., 1995), which is determined by the ability of plant stomata to maintain a diffusive gradient of $\mathrm{CO}_{2}$ concentration from the atmosphere into the cellular structure. Estimates of Archean $\mathrm{CO}_{2}$ have ranged from values close to those of modern Earth to orders of magnitude higher (e.g., Rosing et al., 2010; Dauphas and Kasting, 2011; Driese et al., 2011; Kanzaki and Murakami, 2015); in our previous work (Arney et al., 2016, 2017), we adopted the values from Driese et al. (2011) for our nominal estimates of $\mathrm{pCO}_{2}$ at 2.7 $\mathrm{Ga}\left(\mathrm{pCO}_{2} \sim 1 \times 10^{-3}\right.$ to $1 \times 10^{-2}$ bar). Here, we choose an upper limit on $\mathrm{CO}_{2}$ that is an order of magnitude larger than the Driese et al. (2011) range. Note that organic haze formation in a much more oxidizing atmosphere (such as Marslike with $95 \% \mathrm{CO}_{2}$ or modern day Earth-like with its $21 \%$ $\mathrm{O}_{2}$ ) is not tenable at any plausible hydrocarbon production rates. For significantly more reducing atmospheres than those shown here (i.e., Titan-like), haze formation can be possible at very low methane source fluxes compared to the ones we simulate, which would make an argument for biological involvement in methane production difficult. In this study, methane surface fluxes were chosen to range between $6.8 \times 10^{8}$ to $1 \times 10^{12}$ molecules $/ \mathrm{cm}^{2} / \mathrm{s}$. The lower limit on methane production is taken from the theoretical study of abiotic, serpentinization-driven methane production by Guzmán-Marmolejo et al. (2013) for Earth-like worlds. Life on Earth produces $\mathrm{CH}_{4}$ at a rate of $\sim 1 \times 10^{11}$ molecules $/ \mathrm{cm}^{2} / \mathrm{s}$, and the upper limit for methane flux we consider is an order of magnitude larger than this amount.
For haze optical properties, we use the optical constants of Khare et al. (1984) subject to the caveats outlined in Arney et al. (2016), where we discuss how these optical constants were derived for Titan-analog (not Archeananalog) hazes. However, Archean-simulant haze optical constants have only been measured at one wavelength $(532 \mathrm{~nm})$ by a previous study (Hasenkopf et al., 2010), and the Khare et al. (1984) haze measurements agree reasonably well with the Hasenkopf et al. (2010) measurement. We are currently involved with laboratory work to simulate and measure new optical constants for Archean-analog organic hazes from the UV to the NIR, but haze production rates are slow in $\mathrm{CO}_{2}$-rich conditions, and the analyses will not be ready in time for inclusion in this manuscript. However, we will use these updated optical constants in the future and make them publically available once we do.

We assume a total surface pressure of 1 bar for all simulations. The background atmosphere is composed of $\mathrm{N}_{2}$. When we refer to $\mathrm{CH}_{4} / \mathrm{CO}_{2}$ ratios, note that we are referring to the value at the surface since $\mathrm{CH}_{4}$ does not follow an isoprofile in these atmospheres. $\mathrm{CO}_{2}$, meanwhile, is assumed to be well mixed. We set molecular oxygen $\left(\mathrm{O}_{2}\right)$ at a mixing ratio of $1 \times 10^{-8}$, corresponding to a time after the origin of oxygenic photosynthesis but before substantial oxygen accumulation in the atmosphere (Kharecha et al., 2005; Claire et al., 2014). Note that haze can form at higher oxygen concentrations than considered here and possibly even at oxygen concentrations corresponding to the low Proterozoic $\mathrm{O}_{2}$ levels suggested by Planavsky et al. (2014) of $0.1 \%$ the present atmospheric level as shown by Kurzweil et al. (2013) and Izon et al. (2017). However, haze can only form in such atmospheres at $\mathrm{CH}_{4}$ fluxes higher than those we consider here.

To generate spectra, we use the HITRAN 2012 linelists (Rothman et al., 2013) and set a solar zenith angle of $60^{\circ}$, which approximates the flux observed at quadrature. As in Atmos, optical constants for the haze particles in SMART are derived from the Khare et al. (1984) optical constants using the fractal mean field approximation.

\section{Results}

In this section, we consider haze as a biosignature in the context of atmospheres with and without $\mathrm{S}_{\text {org }}$ gases.

As a baseline case, we first consider haze formation in the absence of biogenic sulfur gases to explore how varying $\mathrm{CO}_{2}$ and $\mathrm{CH}_{4}$ levels affects haze production. Figure 2 shows the optical thickness of an organic haze in the atmosphere of an Archean planet with no $\mathrm{S}_{\text {org }}$ orbiting the ancient $(2.7 \mathrm{Ga})$ Sun, and Fig. 3 shows the same for an Archean-analog planet orbiting GJ 876. In these contour plots, optical depth of unity at $190 \mathrm{~nm}$ (chosen to represent hazes that would significantly impact photochemistry and the planet's spectrum) is marked with the solid black lines overlying the colored contours. Figures 2 and 3 show that lower $\mathrm{CH}_{4}$ fluxes are needed to form optically thick hazes for the simulated planets around GJ 876 compared to the planets simulated around the ancient Sun. This is consistent with our findings in Arney et al. (2017), which showed that lower $\mathrm{CH}_{4} / \mathrm{CO}_{2}$ ratios are required to form hazes around Archeananalog worlds orbiting GJ 876, because this star's lower UV output generates fewer haze-destroying oxygen radicals 


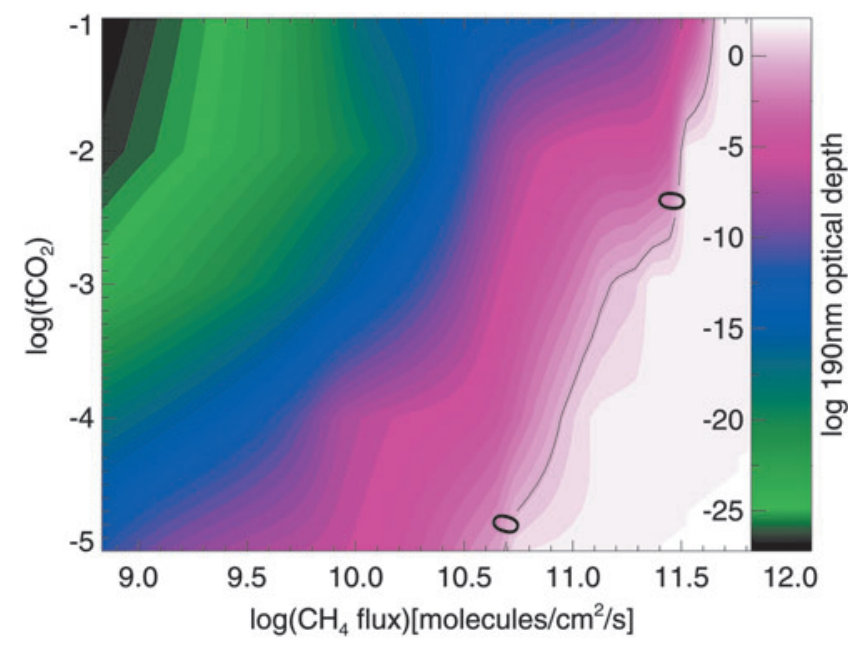

FIG. 2. The colored contours show the log optical depth of the Archean haze (for a planet orbiting the Sun) at $190 \mathrm{~nm}$ as a function of the $\log$ atmospheric fraction of $\mathrm{CO}_{2}$, $\log \left(f \mathrm{CO}_{2}\right)$, on the vertical axis, and the $\log$ of the $\mathrm{CH}_{4}$ surface flux on the horizontal axis. Haze optical depth of unity $(\log (1)=0)$ is marked by the solid black line.

from processes like $\mathrm{CO}_{2}$ photolysis. At the Driese et al. (2011) $\mathrm{CO}_{2}$ levels, haze production requires methane fluxes broadly consistent with estimated Archean biological methane production rates $\left(\sim 0.3-2.5 \times 1 \times 10^{11}\right.$ molecules/ $\mathrm{cm}^{2} / \mathrm{s}$ ) according to Kharecha et al. (2005). This is the case for both simulations of a planet orbiting the Archean Sun and simulations of a planet orbiting GJ 876.

In Figs. 4-7, we include $S_{\text {org }}$ fluxes to show how this additional source of hydrocarbons affects haze formation. Figures 4 and 6 are analogous to Figs. 2 and 3 for the Sun and GJ 876, respectively. In the gray region of Figs. 6 and 7, simulations are not converged; in this part of parameter space, very thick hazes generated extreme stratospheric heating ( $\sim 400 \mathrm{~K})$, causing model instabilities. In Figs. 5 and 7 , we show the same data, but here the horizontal axis shows the $\mathrm{CH}_{4} / \mathrm{CO}_{2}$ ratio instead of the $\mathrm{CH}_{4}$ flux for two

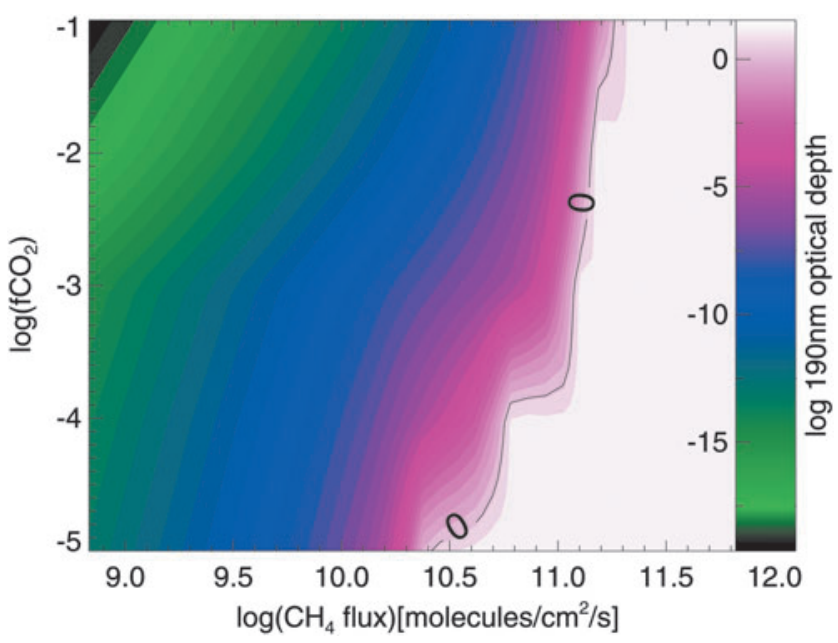

FIG. 3. Same as Fig. 3 but for an Archean-analog planet orbiting GJ 876. reasons. First, this emphasizes how the presence of significant $\mathrm{S}_{\text {org }}$ fluxes can impact the $\mathrm{CH}_{4} / \mathrm{CO}_{2}$ ratio required to form haze. Second, these plots show the actual spectral observables since it is the $\mathrm{CH}_{4}$ mixing ratio-not its fluxthat can be observed from a spectrum (the latter could only be inferred through modeling once the concentrations of atmospheric gases - and the UV spectrum of the star-were measured). In all four figures of simulations including $S_{\text {org }}$ fluxes (Figs. 4-7), the top panel corresponds to the optical depth contours for $1 \times$ the modern $S_{\text {org }}$ fluxes as defined in Domagal-Goldman et al. (2011), and the bottom panel corresponds to $30 \times$ the modern $S_{\text {org }}$ fluxes, chosen for consistency with the upper limit for the $S_{\text {org }}$ flux value in DomagalGoldman et al. (2011). In both panels, the solid red line denotes where the haze's $190 \mathrm{~nm}$ optical depth equals unity for $1 \times S_{\text {org }}$, and the solid black line denotes where the haze optical depth is unity for $30 \times \mathrm{S}_{\text {org }}$. These lines are plotted together in both panels so that they can be easily compared.

Figures 5 and 7 show that as $f \mathrm{CO}_{2}$ decreases, the $\mathrm{CH}_{4} /$ $\mathrm{CO}_{2}$ ratio required to form a thick haze increases. This result is counter-intuitive and arises because the total carbon budget of the atmosphere decreases as $\mathrm{CO}_{2}$ is removed; in other words, any given atmosphere has less $\mathrm{CH}_{4}$ available to form haze at a fixed $\mathrm{CH}_{4} / \mathrm{CO}_{2}$ ratio at lower $\mathrm{CO}_{2}$ levels. More significantly, when fluxes of $\mathrm{S}_{\text {org }}$ increase, the $\mathrm{CH}_{4} /$ $\mathrm{CO}_{2}$ ratio necessary to form a haze decreases at all $\mathrm{CO}_{2}$ levels for planets orbiting both stars. This occurs because photochemistry readily forms methyl groups from organic sulfur gases, and formation of $\mathrm{CH}_{3}$ is a step in the haze formation process. By increasing the efficiency of these processes, the $\mathrm{CH}_{4} / \mathrm{CO}_{2}$ ratio required to form haze at a given $\mathrm{CO}_{2}$ concentration is lowered.

For a solar-type star with $1 \times \mathrm{S}_{\text {org }}$, Fig. 4 shows the $\mathrm{CH}_{4}$ fluxes required to form haze are similar to the simulations with no $\mathrm{S}_{\text {org }}$. However, in the presence of $30 \times \mathrm{S}_{\text {org }}$, the $\mathrm{CH}_{4}$ fluxes required to initiate haze formation decrease by about $50 \%$ for the highest $\mathrm{CO}_{2}$ level considered $\left(f \mathrm{CO}_{2}=10^{-1}\right)$ and by almost an order of magnitude for $f \mathrm{CO}_{2}=10^{-3}$. Figure 5 shows that at $f \mathrm{CO}_{2}=10^{-2}$, the $\mathrm{CH}_{4} / \mathrm{CO}_{2}$ ratio needed to form a haze for the planet around the solar-type star with $\tau$ $(190 \mathrm{~nm})=1$ at $190 \mathrm{~nm}$ is about 0.2 for the $1 \times S_{\text {org }}$ case. This is roughly the same as the $\mathrm{CH}_{4} / \mathrm{CO}_{2}$ ratio required to form a substantial haze in the absence of $\mathrm{S}_{\text {org }}$ (Arney et al., 2016). For the $30 \times \mathrm{S}_{\text {org }}$ case, the $\mathrm{CH}_{4} / \mathrm{CO}_{2}$ ratio required to form substantial haze for the same planet with $f \mathrm{CO}_{2}=10^{-2}$ is about 0.16 , a $20 \%$ decrease.

Around GJ 876, $\mathrm{S}_{\text {org }}$ has a larger impact on haze formation with markedly less methane required to form an optically thick haze at high $\mathrm{S}_{\text {org }}$ fluxes. In Fig. 6, at $f \mathrm{CO}_{2}=10^{-2}$, the haze becomes optically thick at over an order of magnitude smaller $\mathrm{CH}_{4}$ fluxes in the presence of $30 \times \mathrm{S}_{\text {org }}$ compared to $1 \times \mathrm{S}_{\text {org }}$. Figure 7 shows that at $1 \times \mathrm{S}_{\text {org }}$, the haze becomes optically thick at slightly less than $\mathrm{CH}_{4} / \mathrm{CO}_{2}=0.2$, but for $30 \times \mathrm{S}_{\text {org }}$, the haze becomes optically thick at $\mathrm{CH}_{4} /$ $\mathrm{CO}_{2} \sim 0.02$, a full order of magnitude lower. As discussed above, haze forms more readily in the atmosphere of the GJ 876 planet because GJ 876's spectrum results in the production of a smaller quantity of haze-destroying oxygen radicals from $\mathrm{CO}_{2}$ photolysis compared similar planets orbiting the Sun (Arney et al., 2017).

Figures 5 and 7 show that for planets around both stars, as $f \mathrm{CO}_{2}$ decreases (and, therefore, as the absolute amount of 
FIG. 4. The log of the $190 \mathrm{~nm}$ optical depth of organic haze for planets around the Sun at $1 \times \mathrm{S}_{\text {org }}$ and $30 \times \mathrm{S}_{\text {org }}$ as a function of $\log (f$ $\mathrm{CO}_{2}$ ) and surface $\mathrm{CH}_{4}$ flux. The red line in both panels shows where optical depth is unity for $1 \times S_{\text {org }}$, and the black line in both panels shows where optical depth is unity for $30 \times \mathrm{S}_{\text {org }}$. The $1 \times$ and $30 \times \mathrm{S}_{\text {org }}$ optical depth of unity lines are overlain over the contours on both panels so that they may be easily compared.
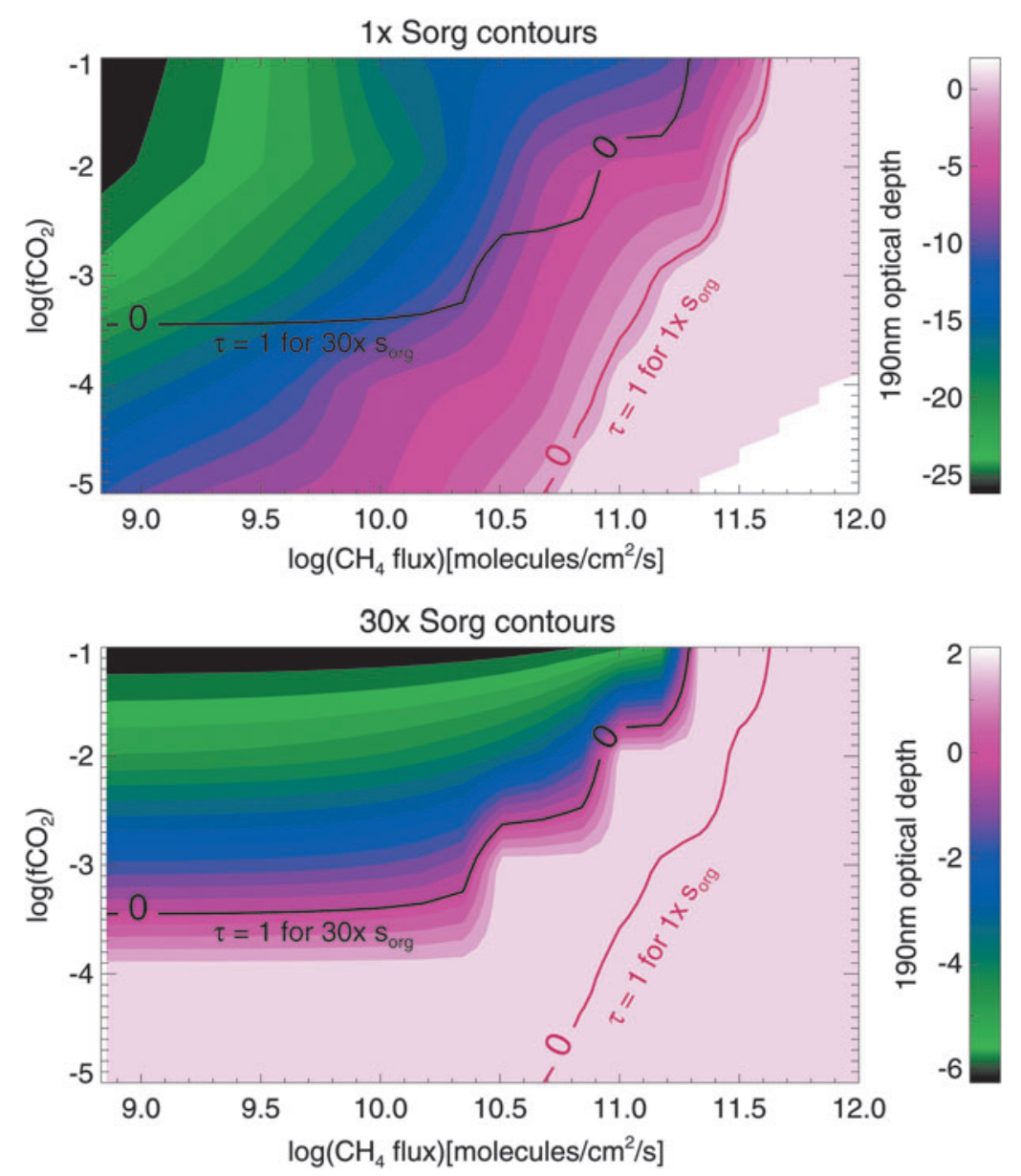

$\mathrm{CH}_{4}$ in the atmosphere at a given $\mathrm{CH}_{4} / \mathrm{CO}_{2}$ ratio also decreases), the difference between the $\mathrm{CH}_{4} / \mathrm{CO}_{2}$ ratios required to form a thick haze with $1 \times S_{\text {org }}$ versus $30 \times S_{\text {org }}$ increases because $S_{\text {org }}$ becomes a larger proportional contributor to the atmosphere's hydrocarbon budget.

Interestingly, $\mathrm{S}_{\text {org }}$ gases themselves provide enough hydrocarbons to not only affect haze formation but also affect the absolute methane abundance. For example, for a planet around the Sun with a methane flux of $1 \times 10^{11}$ molecules/ $\mathrm{cm}^{2} / \mathrm{s}$ and $f \mathrm{CO}_{2}=1 \times 10^{-2}$, we find that an atmosphere without $\mathrm{S}_{\text {org }}$ gases generates a surface methane mixing ratio of $2.2 \times 10^{-4}$, while an atmosphere with $30 \times \mathrm{S}_{\text {org }}$ gases generates a surface methane mixing ratio of $6.8 \times 10^{-4}$. Around GJ 876, the same planets have surface methane mixing ratios of $6.7 \times 10^{-4}$ and $7.3 \times 10^{-3}$ for no $S_{\text {org }}$ and $30 \times$ $\mathrm{S}_{\text {org }}$, respectively. The largest photochemical sources of $\mathrm{CH}_{4}$ in the $30 \times \mathrm{S}_{\text {org }}$ atmospheres are $\mathrm{CH}_{3}+\mathrm{HCO} \rightarrow \mathrm{CH}_{4}+$ $\mathrm{CO}$ and $\mathrm{CH}_{3}+\mathrm{H} \rightarrow \mathrm{CH}_{4}$. These reactions occur 1-2 orders of magnitude faster in the $30 \times \mathrm{S}_{\text {org }}$ atmospheres due to the production of $S_{\text {org }}$-derived methyl radicals. Although the high $S_{\text {org }}$ atmospheres have higher methane levels, which itself allows haze to form more readily, Figs. 5 and 7 show clearly that the hazes in the high- $\mathrm{S}_{\text {org }}$ atmospheres still form at lower methane mixing ratios than they would without $S_{\text {org }}$ gases because the $\mathrm{S}_{\text {org }}$ gases also contribute other gases relevant to haze formation (e.g., $\mathrm{CH}_{3}$ and $\mathrm{C}_{2} \mathrm{H}_{6}$ ). The methane derived from $\mathrm{S}_{\text {org }}$ gases also imposes a lower limit on the amount of methane in these high $S_{\text {org }}$ atmospheres.
For instance, the lowest $\mathrm{CH}_{4} / \mathrm{CO}_{2}$ ratios generated in our $30 \times \mathrm{S}_{\text {org }}$ simulations (bottom panels of Figs. 5 and 7) are higher than the lowest $\mathrm{CH}_{4} / \mathrm{CO}_{2}$ ratios for $1 \times \mathrm{S}_{\text {org }}$ (top panels of Figs. 5 and 7).

\section{Discussion}

Haze formation in atmospheres with Archean-like levels of $\mathrm{CO}_{2}$ can indicate methane production rates consistent with known and theoretical Earth-like biogenic methane production rates, and these fluxes are higher than known and theoretical rates of abiotic methane production on modern Earth. However, more efficient abiotic methane production rates on early Earth and exoplanets cannot be ruled out. Thus, while haze would not be definitive proof of life on these planets, it would indicate that they are consistent with the behavior of Earth's methanogenic biosphere and therefore are highly interesting targets for follow-on studies. We have shown here that detecting an organic haze in the presence of a relatively low $\mathrm{CH}_{4} / \mathrm{CO}_{2}$ ratio could further imply the influence of biogenic sulfur gases aiding haze formation. This is similar to the suggestion of ethane as a spectral biosignature in Domagal-Goldman et al. (2011), as photolysis of methyl-bearing $S_{\text {org }}$ gases enhances formation of ethane in atmospheres with large $S_{\text {org }}$ fluxes even if the $S_{\text {org }}$ gases themselves are difficult to detect directly. The impact of $S_{\text {org }}$ on haze formation is more pronounced around M dwarf stars like GJ 876 because the lower overall UV flux 

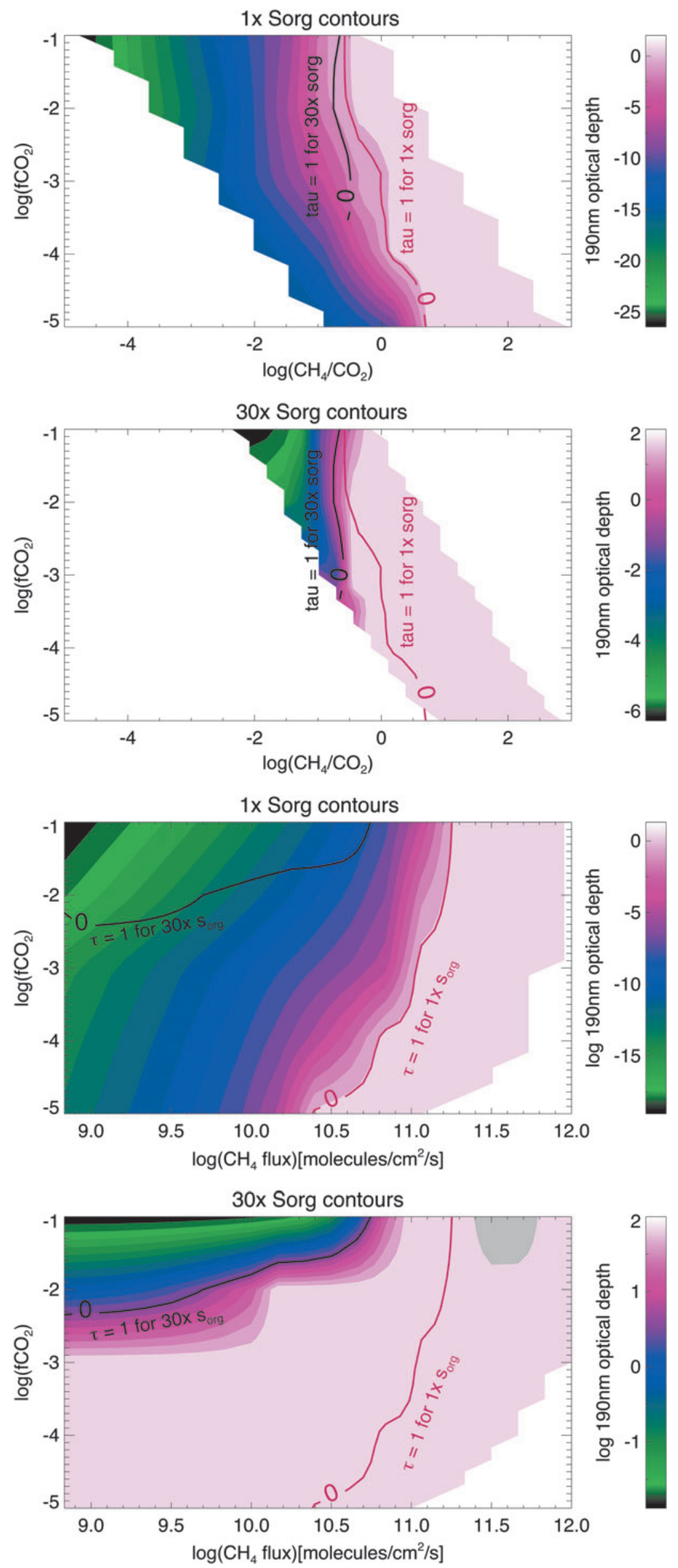

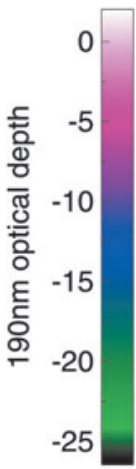

FIG. 5. Same as Fig. 4 but showing the log of the $190 \mathrm{~nm}$ optical depth of organic haze for planets around the Sun at $1 \times \mathrm{S}_{\text {org }}$ and $30 \times \mathrm{S}_{\text {org }}$ as a function of $\log \left(f \mathrm{CO}_{2}\right)$ and $\log \left(\mathrm{CH}_{4} / \mathrm{CO}_{2}\right)$.

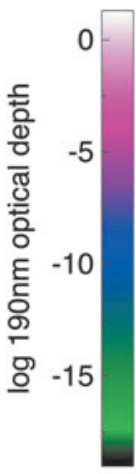

FIG. 6. Same as Fig. 4 but for planets orbiting GJ 876. Simulations in the gray region are not converged. 
FIG. 7. Same as Fig. 5 but for planets orbiting GJ 876.
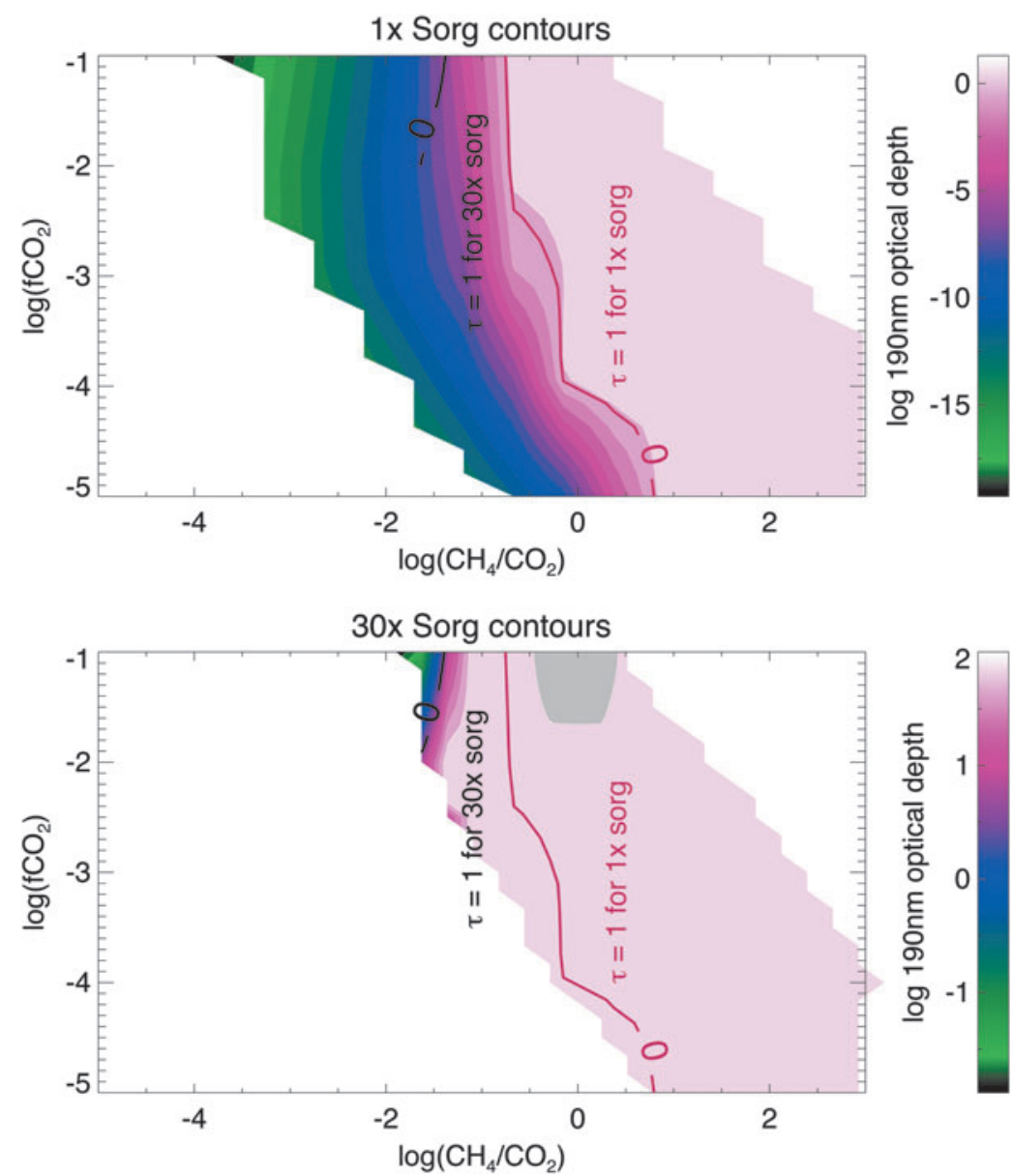

around this star leads to smaller sinks of hydrocarbon gases (Domagal-Goldman et al., 2011; Arney et al., 2017).

In all situations - including planets without $S_{\text {org }}$ gases-it will be important to know the redox state and temperature regime of an atmosphere to argue for the plausible biogenicity of an organic haze. Titan shows us that abiotic hazes can form in highly reducing cold atmospheres with long residence lifetimes for methane. Estimating the temperature of a planet will require measurements of the planet's semimajor axis and greenhouse gas budget. However, the temperature difference between Titan and early Earth is large - about $200 \mathrm{~K}$ - and so only broad temperature constraints would be needed to separate Titan-like planets from Earth-like planets. To understand the atmospheric redox state of a given world, measurements of $\mathrm{CO}_{2}$ and $\mathrm{CH}_{4}$ will be required to constrain the $\mathrm{CH}_{4} / \mathrm{CO}_{2}$ ratio. Additional discussion on the interpretation of a haze spectral feature in the context of the rest of the planetary environment can be found in Section 4.3.

\subsection{Detectability considerations}

Transit observations with JWST (Beichman et al., 2014) could provide access to NIR wavelengths on hazy exoEarths and were discussed in detail by Arney et al. (2017). Planets orbiting $\mathrm{M}$ dwarfs are more amenable to observations with JWST compared to planets around solar-type stars due to the larger planet-to-star size ratio and more frequent transits for planets in the habitable zone. In Fig. 8 we show the transit transmission spectra of hazy Archean Earth with $1 \times$ and $30 \times S_{\text {org }}$ around the Sun and GJ 876 to illustrate which spectral features may be detectable for these worlds. The planets shown around the Sun-like star were simulated with a methane flux of $6.9 \times 10^{10}$ molecules $/ \mathrm{cm}^{2} / \mathrm{s}$ and a $\mathrm{CO}_{2}$ mixing ratio of $1 \times 10^{-3}$, corresponding to surface methane mixing ratios of $7.9 \times 10^{-5}$ and $3.2 \times 10^{-4}$ for $1 \times$ and $30 \times \mathrm{S}_{\mathrm{org}}$, respectively. The planets around the GJ 876-like star were simulated with a methane flux of $3.1 \times 10^{10}$ molecules $/ \mathrm{cm}^{2} / \mathrm{s}$ and a $\mathrm{CO}_{2}$ mixing ratio of $1 \times 10^{-2}$, with resultant surface methane mixing ratios of $1.4 \times 10^{-4}$ and $1.2 \times 10^{-3}$. These atmospheres were chosen to represent cases where haze is not significantly present at $1 \times S_{\text {org }}$ but is present at $30 \times \mathrm{S}_{\text {org }}$. As expected, Fig. 8 shows that the spectral consequences of high $\mathrm{S}_{\text {org }}$ fluxes are more significant for the planet orbiting GJ 876.

Even at $30 \times \mathrm{S}_{\text {org }}$, organic sulfur gases are not apparent in the transit transmission spectrum. Domagal-Goldman et al. (2011) showed that these $S_{\text {org }}$ gases are concentrated in the lower atmosphere since they are readily photolyzed at higher layers, so they are spectrally inaccessible at the altitudes probed by transits for a hazy planet $(20-80 \mathrm{~km}$ for the planets in Fig. 8). Haze, $\mathrm{CH}_{4}, \mathrm{CO}_{2}$, and $\mathrm{C}_{2} \mathrm{H}_{6}$ are all potentially detectable, however. Organic haze could be discerned through the presence of a haze-induced NIR spectral slope, and haze produces an absorption feature near $6 \mu \mathrm{m}$ and a much weaker one near $3 \mu \mathrm{m}$ (not labeled). $\mathrm{CH}_{4}$ absorbs near 1.1, 1.4, 1.7, 

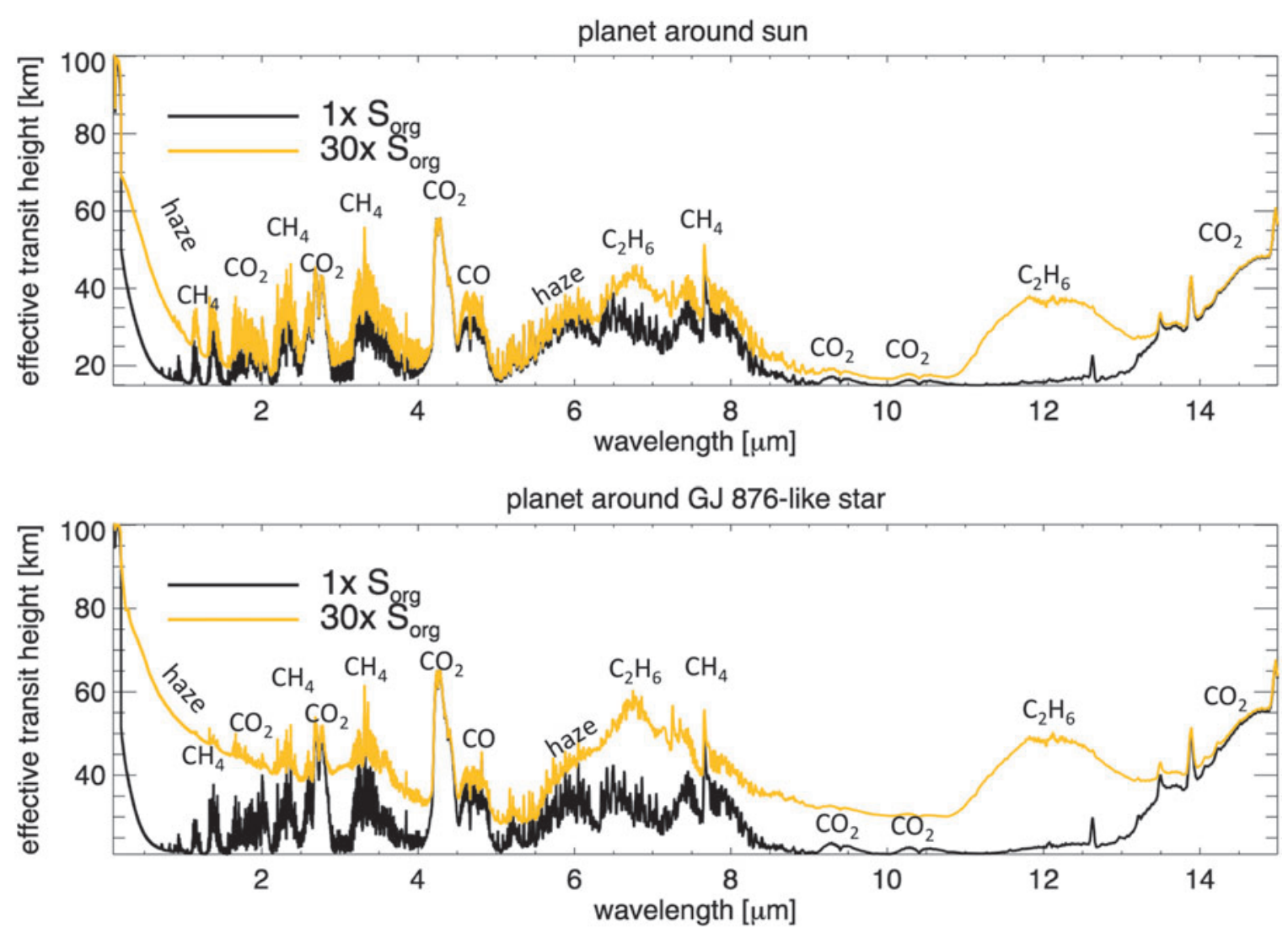

FIG. 8. Representative transit transmission spectra of Archean Earth-like planes with different $\mathrm{S}_{\text {org }}$ fluxes. The $y$ axis shows the effective tangent height, which is the altitude above the planet's surface that light on tangent transit path lengths can penetrate into the atmosphere. Spectra are shown for $\Delta \lambda=0.01 \mu \mathrm{m}$.

2.3, 3.3, and $7.5 \mu \mathrm{m} . \mathrm{CO}_{2}$ also absorbs at wavelengths accessible to JWST at $1.57,2,2.7$, and $4.3 \mu \mathrm{m}$. $\mathrm{C}_{2} \mathrm{H}_{6}$ produces features near 6.5 and $12 \mu \mathrm{m}$. The features at wavelengths longward of about $8 \mu \mathrm{m}$ can probably be considered inaccessible to JWST due to the dim stellar blackbody at these wavelengths. See Arney et al. (2017) for our discussion of how observable these features are with JWST for a planet orbiting a star like GJ 876.

Potential direct imaging telescopes under consideration such as LUVOIR and HabEx may provide direct observations of Earth-like exoplanets in the 2030s and beyond (Postman et al., 2010; Bolcar et al., 2015; Dalcanton et al., 2015; Stapelfeldt et al., 2015; Mennesson et al., 2016). Figure 9 shows the reflected light spectra of the same planets shown in Fig. 8. Haze produces a broad, deep spectral feature at UV-blue wavelengths; for this combination of $\mathrm{CH}_{4}$ $\mathrm{CO}_{2}-\mathrm{S}_{\text {org }}$, the haze feature is weaker for the planet around the Sun-like star compared to the planet around the GJ 876like star, but it still significantly alters the shape of the spectrum at wavelengths $<0.5 \mu \mathrm{m}$. This very strong feature is the reason why we argue here that haze is likely a much more detectable indicator of atmospheres with $\mathrm{S}_{\text {org }}$ compared to the $\mathrm{C}_{2} \mathrm{H}_{6}$ discussed in Domagal-Goldman et al. (2011) that absorbs in the mid-IR. Of course, for both transit transmission and direct imaging observations, how well we will be able to determine whether a haze is present at an anomalously low $\mathrm{CH}_{4} / \mathrm{CO}_{2}$ ratio depends on how well we will be able to retrieve the $\mathrm{CH}_{4}$ and $\mathrm{CO}_{2}$ gas concentrations.
To examine the spectral resolutions $(R=\lambda / \Delta \lambda)$ required to observe features in hazy reflected light spectra, Fig. 10 shows the reflectance spectrum of a representative hazy Archean Earth at several spectral resolutions for $\mathrm{COO}_{2}=10^{-2}$ and $\mathrm{CH}_{4} /$ $\mathrm{CO}_{2}=0.2$. The Fig. 10 spectrum includes water clouds added to our $1 \mathrm{D}$ radiative transfer model by using a weighted averaging technique where $50 \%$ of the planet is considered haze-covered and cloud-free, $25 \%$ is covered by haze and stratocumulus clouds, and $25 \%$ is covered by cirrus clouds and haze (Robinson et al., 2011). Haze produces a broad, strong absorption feature at short wavelengths that can be easily resolved at spectral resolutions as low as 10 . Methane and $\mathrm{CO}_{2}$ are observable in the NIR, although $\mathrm{CO}_{2}$ is more challenging to detect. Carbon dioxide has features near 1.57 and $2 \mu \mathrm{m}$ for the wavelengths shown here, but telescope thermal emission could swamp the $2 \mu \mathrm{m}$ feature for a noncryogenically cooled mirror. Therefore, the most detectable $\mathrm{CO}_{2}$ feature in reflected light occurs at $1.57 \mu \mathrm{m}$ for Archean-like $\mathrm{CO}_{2}$ levels. Resolving the narrow multipeaked structures of the $1.57 \mu \mathrm{m} \mathrm{CO}_{2}$ feature will require spectral resolution $R>100$, and $R>170$ will be required to correctly resolve the depths of the narrow bands of this feature. As in the transit transmission spectrum, $S_{\text {org }}$ features are not detectable in the reflected light spectrum.

Although there are numerous interesting spectral features in the NIR, they may not be detectable in direct imaging observations even if telescopic thermal emission is negligible because inner working angle (IWA) constraints alone can limit access to longer wavelengths. The IWA represents the smallest 

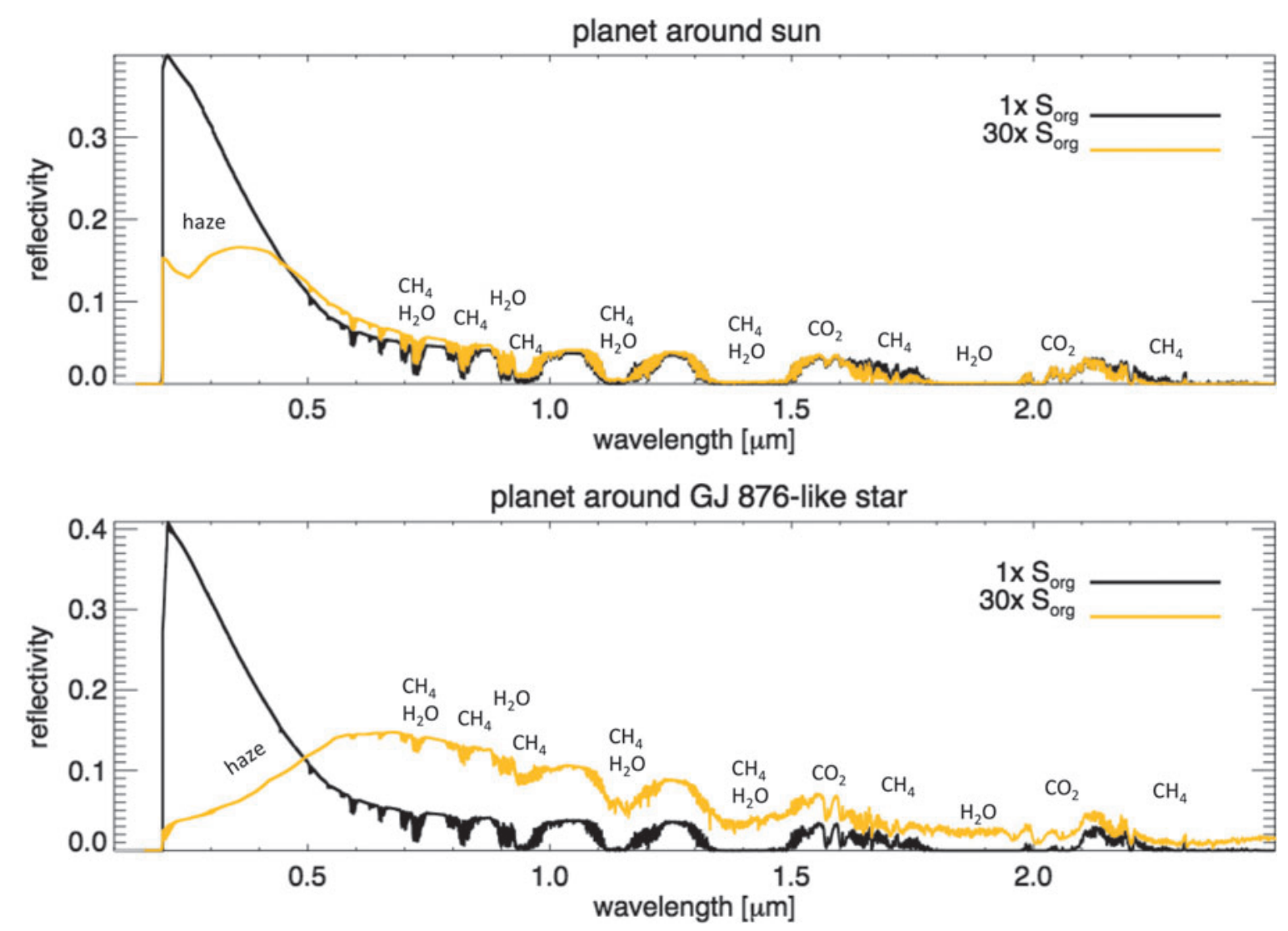

FIG. 9. Representative reflected light spectra of Archean Earth-like planes with different $S_{\text {org }}$ amounts for the same atmospheres shown in Fig. 8. These spectra do not include water clouds to show the spectral impact of only organic haze.

planet-star angular separation that can be resolved with sufficient signal-to-noise to detect the planet. For a coronagraph, the IWA scales with $C \times \lambda / D$, where $C$ is a small valued-constant of order unity and $D$ is the telescope diameter. Several mirror diameters are being considered for the designs of the HabEx and LUVOIR mission concepts. Currently, these designs include a $4 \mathrm{~m}$ monolith (HabEx), a JWST-sized $6.5 \mathrm{~m}$ mirror (HabEx), a $9.2 \mathrm{~m}$ mirror (LUVOIR), and a $15.1 \mathrm{~m}$ mirror (LUVOIR). Note, however, that light reflected off the jagged hexagonal segments on the outside of a segmented mirror poses a challenge for current coronagraph designs. Therefore, to be conservative, we will consider only light reflected from the largest inscribed circle that fits within each segmented aperture, resulting in a smaller effective aperture visible to the coronagraph. For the segmented telescope sizes listed previously, these correspond to inscribed diameters of 5.5, 7.6, and $12.7 \mathrm{~m}$, respectively, for the current designs of these telescopes (M. Bolcar, personal communication).

To receive an Archean-like level of instellation (i.e., stellar irradiation), a planet orbiting a star like GJ 876 would be at a planet-star separation distance of 0.12 AU. Assuming an optimistic IWA of $\lambda / D$ and a $12.7 \mathrm{~m}$ aperture, a GJ 876like planet-star system could be located no farther away than $4.5 \mathrm{pc}$ for the IWA to allow characterization of the spectrum out to $1.57 \mu \mathrm{m}$ to see the $\mathrm{CO}_{2}$ band using a coronagraph. Smaller telescopes fare worse, as does assuming more conservative IWAs such as IWA $=2 \lambda / D$ or IWA $=3 \lambda / D$. Table 1 shows the longest wavelength observable before the IWA cuts off the spectrum for planet-star systems located at a fixed distance of $4.5 \mathrm{pc}$ assuming a solar-type star and a GJ 876-type star for the four different telescope sizes being studied by HabEx and LUVOIR (with the IWAs for the segmented telescopes use their inscribed circle diameters). At $4.5 \mathrm{pc}$, it is difficult to characterize the planet orbiting GJ 876 in the NIR unless the telescope is the largest we simulate and/or the IWA is the most optimistic we consider. The influence of $\mathrm{S}_{\text {org }}$ on haze formation is most pronounced around $\mathrm{M}$ dwarfs like GJ 876, but as we have seen, IWA constraints make Earth-like planets around such stars more challenging to characterize since they orbit so close to their host stars. Note, however, that here we are assuming that starlight suppression is achieved with a coronagraph, but star shades may be able to provide smaller IWAs than those discussed here, depending on star-shade size and star shadetelescope separation distance (Turnbull et al., 2012).

Figure 11 shows the integration times required to obtain a signal-to-noise ratio (SNR) of 20 as a function of wavelength for planets with the same hazy Archean atmospheric parameters used to generate Fig. 10 by using the Robinson et al. (2016) coronagraph noise model. We assume $R=170$ in the visible and NIR to fully resolve the structure of the narrow $1.57 \mu \mathrm{m} \mathrm{CO}_{2}$ band peaks and troughs, and $R=20$ in the UV (for $\lambda<0.4 \mu \mathrm{m}$ ). Vertical lines indicate IWA cutoffs as described in the figure caption. We again assume apertures of 4, 5.5, 7.6, and $12.7 \mathrm{~m}$, and the planet-star system is assumed to be $4.5 \mathrm{pc}$ to allow detection of the $1.57 \mu \mathrm{m} \mathrm{CO}_{2}$ band for a planet orbiting a star like GJ 876 with IWA $=\lambda / D$ for the largest telescope. We chose to simulate $\mathrm{SNR}=20$ 

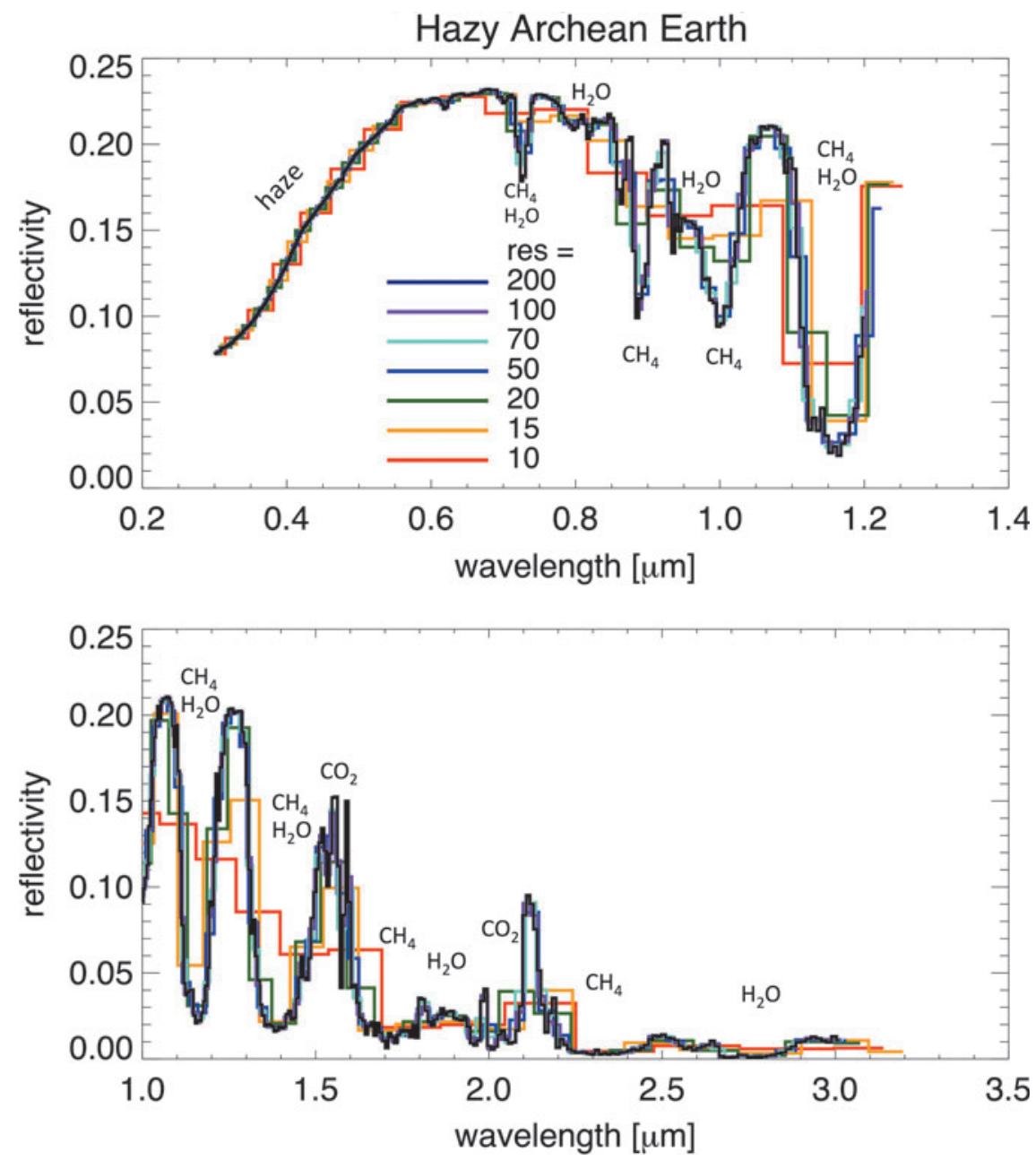

FIG. 10. The reflected light spectra of hazy Archean Earth at several spectroscopic resolutions. The haze absorption feature at $\lambda<0.6 \mu \mathrm{m}$ is sufficiently strong and broad to be resolved at very low spectral resolution. These spectra include water clouds in addition to haze, via a weighted averaging technique in our $1 \mathrm{D}$ model $(50 \%$ haze only, $25 \%$ haze and cirrus cloud, and $25 \%$ haze and stratocumulus cloud). because studies have shown that continuum SNR measurements of about 20 may be required to constrain gas abundances in planetary atmospheres (Lupu et al., 2016; Nayak et al., 2017; M. Marley, personal communication). Note in Fig. 11 that absorption bands, where the planet is darker, require longer integration times to reach $\mathrm{SNR}=20$, but the gas retrieval demands discussed above apply to the continuum regions, where the planet is brighter. Because $\mathbf{M}$ dwarfs are dimmer than Sun-like stars in the visible, longer integration times are required to characterize the planet around GJ 876 at wavelengths $<1 \mu \mathrm{m}$ compared to the planet orbiting the Sun. The situation is reversed for wavelengths $>1 \mu \mathrm{m}$. The dramatic increase in integration times near $1.6 \mu \mathrm{m}$ is caused by the telescope's thermal emission (the mirror is $270 \mathrm{~K}$ ), rendering these longer wavelengths ef- fectively unobservable for a noncryogenic telescope even if they were accessible within the IWA wavelength cutoff.

Integration times at all wavelengths increase by at least an order of magnitude for the $4 \mathrm{~m}$ telescope compared to the $12.7 \mathrm{~m}$ telescope. For the $12.7 \mathrm{~m}$ telescope, sensing continuum regions to a level of $\mathrm{SNR}=20$ would generally require tens to hundreds of hours of observing time for the resolution shown here. For the $4 \mathrm{~m}$ telescope, measuring the continuum at $\mathrm{SNR}=20$ demands hundreds to thousands of hours of integration.

\subsection{Other hydrocarbon-containing gases}

Although we included just $\mathrm{S}_{\text {org }}$ gases in our photochemical model, there are numerous other methyl-containing

Table 1. Wavelength Cutoffs for Four Mirror Diameters and Three IWAs for Planet-Star Systems at 4.5 pC

\begin{tabular}{|c|c|c|c|c|c|c|}
\hline \multirow{2}{*}{$\begin{array}{l}\text { Mirror } \\
\text { diameter }(m)\end{array}$} & \multicolumn{2}{|c|}{ Cutoff for $I W A=\lambda / \mathrm{D}(\mu m)$} & \multicolumn{2}{|c|}{ Cutoff for IWA $=2 \lambda / \mathrm{D}(\mu \mathrm{m})$} & \multicolumn{2}{|c|}{ Cutoff for $I W A=3 \lambda / \mathrm{D}(\mu \mathrm{m})$} \\
\hline & GJ 876 & Sun & GJ 876 & Sun & GJ 876 & Sun \\
\hline 12.7 & 1.6 & 14 & 0.82 & 6.8 & 0.54 & 4.6 \\
\hline 7.6 & 0.98 & 8.2 & 0.49 & 4.1 & 0.33 & 2.7 \\
\hline 5.5 & 0.71 & 6.0 & 0.36 & 3.0 & 0.24 & 2.0 \\
\hline 4 & 0.52 & 4.3 & 0.26 & 2.2 & 0.17 & 1.4 \\
\hline
\end{tabular}


FIG. 11. The wavelength-dependent integration time required to obtain a $\mathrm{SNR}=$ 20 for hazy Archean-analog planets orbiting the Sun and GJ 876 at $4.5 \mathrm{pc}$ as observed by four different telescope architectures. The red, green, and blue vertical lines represent the wavelength cutoffs for IWA $=\lambda / D, 2 \lambda / D$, and $3 \lambda / D$, respectively. The dashed lines are the IWA cutoffs for a GJ 876-like star, and the solid lines represent the IWA cutoffs for a solartype star. Not all IWA cutoffs are shown on every plot if they do not overlap with the wavelength range displayed: for instance, all the solar IWA lines occur redward of the right axis of the 12.7 and $7.6 \mathrm{~m}$ plots. The discontinuity at 0.4 and $0.85 \mu \mathrm{m}$ is due to the boundary between the assumed UV, visible, and NIR detectors.

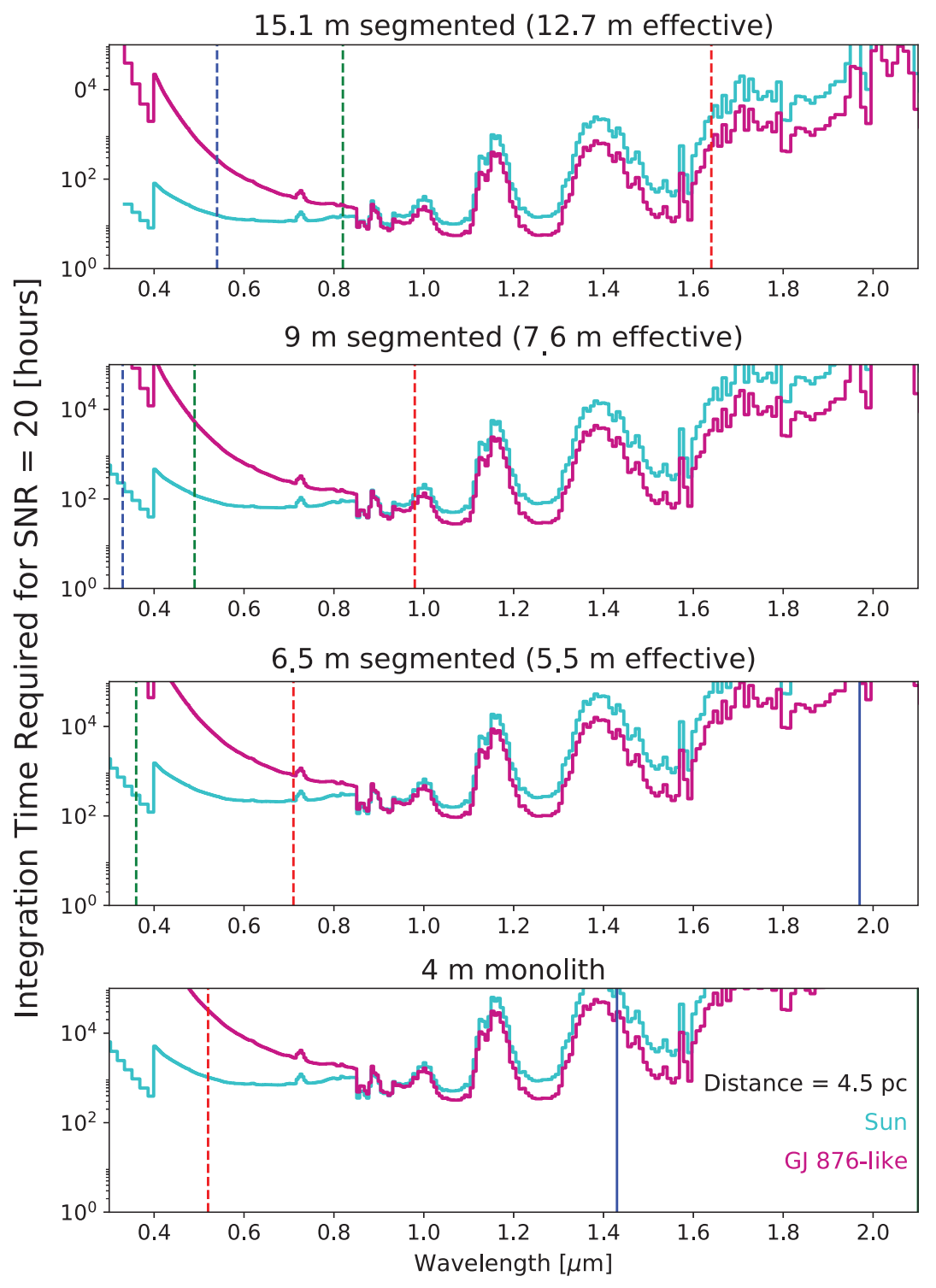

gases produced by terrestrial organisms and other processes (Seager et al., 2012) that may contribute to photochemical organic haze production if present in a planet's atmosphere. Such gases include $\mathrm{CH}_{3} \mathrm{Cl}$ (methyl chloride), $\mathrm{CH}_{3} \mathrm{Br}$ (methyl bromide), $\mathrm{CH}_{3} \mathrm{I}$ (methyl iodide), $\mathrm{CH}_{3} \mathrm{OH}$ (methanol), and terpenes. Methyl chloride, in particular, has been shown in a previous study to have a longer photochemical lifetime in the atmospheres of Earth-like planets orbiting $M$ dwarfs compared to Sun-like stars (Segura et al., 2005), though note that the other methyl-containing compounds listed here were not tested in the Segura et al. study. Natural sources of methyl chloride, the most abundant halocarbon in the atmosphere (Yokouchi et al., 2000), include oceanic sources (Koppmann et al., 1993) such as planktonic algae (Harper et al., 2003), plants (Yokouchi et al., 2000; Rhew, 2011), and biomass burning (Blake et al., 1996). Higher-order halogenated organic compounds such as $\mathrm{C}_{2} \mathrm{H}_{4} \mathrm{Cl}, \mathrm{C}_{3} \mathrm{H}_{7} \mathrm{Cl}$, and $\mathrm{C}_{4} \mathrm{H}_{9} \mathrm{Cl}$, as well as methyl chloride itself, can also be produced in soils and sediments when chlorine ions are alkylated through organic matter oxidation (Keppler et al.,
2000). An overview of methyl chloride sources, including industrial ones, is provided in Keppler et al. (2005). Nonanthropogenic sources of methyl bromide include marine algae and biomass burning (Blake et al., 1996; McCauley et al., 1999), kelp (Manley and Dastoor, 1988), other oceanic sources (Anbar et al., 1996), and plants (Rhew, 2011). Methyl iodide can be produced by vegetation and soils (Sive et al., 2007), oceanic sources (Rasmussen et al., 1982) including kelp and microbial metabolisms (Manley and Dastoor, 1988), and biomass burning (Blake et al., 1996). Methanol is the simplest alcohol and is produced by a variety of anaerobic metabolisms including methanotrophy (Xin et al., 2004). In the atmosphere, it can contribute to the tropospheric HOx budget after being oxidized to formaldehyde (Solomon et al., 2005). Terpenes are a broad class of organic compounds released by plants and insects (Pare and Tumlinson, 1999) that are responsible for atmospheric phenomena such as the low-lying blue haze that can be seen over some forested regions like the Blue Ridge Mountains (Went, 1960). Some terpenes such as d-limonene can 
ultimately generate gases like formaldehyde and formic acid in the atmosphere through photochemical processing (Walser et al., 2007).

The largest potential fluxes of these gases would likely be on planets where they are the by-product of metabolism. On modern Earth, major sources of these gases (including $\mathrm{CH}_{3} \mathrm{SH}$ ) are typically the degradation of amino acids (Stoner et al., 1994; Lomans et al., 2002). However, on planets with a different atmospheric composition, there might be an energetic incentive to produce these gases directly. The potential for this has been demonstrated in the laboratory, in experiments where methanogens are given $\mathrm{H}_{2} \mathrm{~S}$ as a substrate in place of $\mathrm{H}_{2}$, and in response produce $\mathrm{CH}_{3} \mathrm{SH}$ instead of $\mathrm{CH}_{4}$ (Moran et al., 2008). On a planet with globally high $\mathrm{H}_{2} \mathrm{~S}$ concentrations, microbes could release high fluxes of $\mathrm{CH}_{3} \mathrm{SH}$ to the atmosphere, accumulating $\mathrm{CH}_{3} \mathrm{SH}$ and its photochemical by-products $\left(\mathrm{C}_{2} \mathrm{H}_{6}\right.$ in particular) to potentially detectable levels (Domagal-Goldman et al., 2011). Other atmospheric compositions may also lead to an incentive for other gases to be produced. For example, an atmosphere rich in halogens - and $\mathrm{HCl}, \mathrm{HI}$, and $\mathrm{HBr}$ in particular-may incentivize the biological production of $\mathrm{CH}_{3} \mathrm{Cl}, \mathrm{CH}_{3} \mathrm{I}$, and $\mathrm{CH}_{3} \mathrm{Br}$. While this has not been studied in the laboratory, nor modeled in a photochemical simulation, this sort of atmosphere would create the largest concentration of - and therefore spectral signal from-these gases.

Such gases have been considered in the context of atmospheric biosignatures since they are produced by biological processes (Seager et al., 2012), although as we have seen here and in Domagal-Goldman et al. (2011), photochemistry and the strength of these gases' spectral signatures will determine whether they-or any of their photochemical by-products-are useful gases (or aerosols) to target in the search for life beyond Earth. For instance, the spectral signatures of $\mathrm{CH}_{3} \mathrm{Cl}$ were investigated in Segura et al. (2005) in simulations that included photochemistry. $\mathrm{CH}_{3} \mathrm{Cl}$ was readily destroyed by the solar spectrum for a planet orbiting the Sun, but for planets orbiting $\mathrm{M}$ dwarfs $\mathrm{AD}$ Leo or $\mathrm{GJ} 463 \mathrm{C}$, the $\mathrm{CH}_{3} \mathrm{Cl}$ vertical mixing ratio profiles approximated isoprofiles up to at least $70 \mathrm{~km}$ in altitude. For such atmospheres, Segura et al. (2005) showed that $\mathrm{CH}_{3} \mathrm{Cl}$ produces absorption features in the thermal IR near 7, 10, and $15 \mu \mathrm{m}$. Although Segura et al. (2005) did not simulate transit transmission spectra, the well-mixed profiles of $\mathrm{CH}_{3} \mathrm{Cl}$ for the $\mathrm{M}$ dwarfs suggest it may be detectable in transit observations, even if such observations could only probe the upper layers of these atmospheres.

\subsection{Interpretation of biosignatures in the planetary context}

Generally speaking, it is important to consider any potential biosignature in the broader planetary context to rule out false-positive abiotic production mechanisms and search for other signs of habitability and life. Such additional constraints are important in all situations: the best understanding of any planet will come from a holistic consideration of many diverse pieces of information. The Solar System shows us that there is tremendous value in obtaining as much information as possible when interpreting difficult-to-access planetary characteristics. For example, the case for Europa's subsurface ocean was supported and strengthened by multiple lines of evidence ranging from surface morphology to Europa's induced magnetic field (e.g., Squyres et al., 1983; Carr et al., 1998; Kivelson et al., 2000; Stevenson, 2000).

Alarmingly, the nearby worlds in our solar system also have a rich history of misinterpretation in the absence of robust data. Percival Lowell's extensive analysis of the putative (and wholly illusionary) canals on Mars is perhaps the bestknown example of this phenomenon (Lowell, 1906), but there are countless other less notorious cases. For instance, when G. Kuiper discovered Titan's atmosphere (Kuiper, 1944), he speculated that the moon's orange coloration is due to "the action of the atmosphere on the surface itself, analogous to the oxidation supposed to be responsible for the orange color of Mars," not understanding that he was seeing the atmosphere rather than the planetary surface. Exoplanets, spatially unresolved and at vast distances, will be even more difficult to interpret. Because the designs of the first generation of observatories that include terrestrial exoplanet characterization and biosignature detection in their science goals are currently being studied, it is crucial that these design studies be informed by ongoing analyses of a variety of possible biosignatures, habitability signatures, and false positives of both. For these future observatories, a wide wavelength spectral range can mitigate the possibility of reaching erroneous conclusions by providing additional spectral context to consider any given feature. Additional context will be even more valuable when interpreting newly discovered planet types and characteristics not represented in the Solar System (e.g. hot Jupiters, mini-Neptunes, and super Earths). The diversity of planet types already discovered suggests that the probability of detecting a true Earth twin is very small, so it is crucial to expand our understanding of how habitability and biosignatures may appear on worlds different from the planet we live on. Somewhat paradoxically, we must do this before these exoplanets are even discovered; otherwise, we risk underdesigning the capabilities of the future observatories that will study them.

Organic haze is just one example of a potential novel biosignature. Haze itself is relatively simple to detect due to its strong spectral features, but as we have argued here, interpretation of haze spectral features will be challenging, dependent on several different pieces of information. Indeed, Titan's abiotic organic haze shows plainly that the detection of haze alone is inadequate for biosignature considerations. We have, in this study, discussed observing strategies to strengthen the case for a haze as biogenic: (1) When a haze is present in a sufficiently oxidizing background atmosphere, high methane fluxes may be required to produce it, and such high fluxes may suggest (but not prove) the involvement of biology, the most vigorous producer of methane on Earth today. (2) When a haze is present in an atmosphere that has less methane than photochemical models predict is required to initiate haze formation, this implies the existence of an additional hydrocarbon source like $\mathrm{S}_{\text {org }}$ gases. Beyond these two strategies, other measurements like the presence (or absence) of other biosignatures and habitability signatures like ocean glint (Robinson et al., $2010,2014)$ would provide valuable additional information that could strengthen (or weaken) the case for biological involvement.

Future work is necessary to more rigorously examine the rates of abiotic methane production that may be possible on 
other planet types and determine whether there are other abiotic mechanisms that generate hazes at unexpectedly low $\mathrm{CH}_{4} / \mathrm{CO}_{2}$ ratios. Additionally, as we emphasized above, future studies of other novel biosignatures and habitability signatures are also needed to expand our palette of known possible types of living planets. However, because photochemistry may generate non-intuitive or indirect spectral signs of these biological processes (e.g., the spectral impacts of $S_{\text {org }}$ ), it is crucial for any considerations of novel spectral signatures to also include the context of the broader atmospheric and stellar flux environment.

\subsection{False positives for organic haze}

We considered several possible spectral mimics for organic haze in our previous study (Arney et al., 2017). Given that the most detectable spectral signature of organic haze is its blue-UV absorption feature, other compounds with strong UV-blue absorption have the potential to be mistaken for organic haze. These include iron oxide, the unknown UV absorber in the venusian atmosphere, and exotic haze compounds such as zinc sulfide ( $\mathrm{ZnS}$, although its high condensation temperature means it is not a viable aerosol candidate for Earth-like atmospheres). An additional UVblue-absorbing compound we did not consider in our previous study is $S_{8}$ particles, which can produce deep, broad blue-UV absorption features similar to organic haze when present in large quantities (Hu et al., 2013). $\mathrm{S}_{8}$ particles are produced by volcanic sulfur gas emissions; photochemical processing of these volcanic gases tends to favor production of $\mathrm{H}_{2} \mathrm{SO}_{4}$ aerosols under more oxidizing conditions, while $\mathrm{S}_{8}$ is favored under more reducing conditions such as those that would also tend to generate organic haze (e.g., Zahnle et al, 2006; Hu et al., 2013). It may be possible to distinguish the spectral signatures of $S_{8}$ from organic haze via absorption features from emitted volcanic gases themselves (e.g., $\mathrm{H}_{2} \mathrm{~S}$ and $\mathrm{SO}_{2}$ ), but modeling work by $\mathrm{Hu}$ et al. (2013) shows these gases are photochemically short-lived, and their most detectable absorption features occur longward of $5 \mu \mathrm{m}$. This makes direct imaging detections of them very difficultalthough transit observations probing to longer wavelengths may still be able to measure them. Organic haze produces its own diagnostic absorption features near 3 and $6 \mu \mathrm{m}$ that may also be detectable in transit observations, allowing it to be distinguished from $\mathrm{S}_{8}$ if IR transit observations are possible. We emphasize, again, as we did in Section 4.3, that any given spectral feature must be considered in the context of the whole planetary environment and with as much spectral contextual information as possible. A strong UV-blue wavelength absorber is more likely to be organic haze than $\mathrm{S}_{8}$ particles if it is detected in the presence of strong $\mathrm{CH}_{4}$ features, but if $\mathrm{CH}_{4}$ features are weak or absent, $\mathrm{S}_{8}$ (or other compounds like iron oxide) may be more likely. $S_{8}$ (and sulfate) concentrations may also be time-variable if volcanic outbursts occur sporadically, and time-resolved spectroscopy may be a powerful means of identifying false positives of volcanic emissions (Misra et al., 2015).

\section{Conclusions}

Organic haze formation on Archean Earth was likely controlled by biological methane production, and this type of haze may also occur on anoxic worlds elsewhere. On planets with Archean-like $\mathrm{CO}_{2}$ levels, organic haze formation requires methane production consistent with known and theoretical biological fluxes on Earth. However, because abiotic processes can also produce methane, methods to distinguish biotic and abiotic hazes are needed. To that end, we explored how biogenic organic sulfur gases affect haze formation since these gases can liberate methyl radicals that become involved in haze production. We find organic sulfur gases can drive haze formation at lower $\mathrm{CH}_{4} / \mathrm{CO}_{2}$ ratios compared to methane photochemistry alone. This effect is especially pronounced around M dwarfs with lower UV fluxes than the Sun. To make the case for a $S_{\text {org }}$-mediated haze impacting a planet's spectrum, it will be necessary to constrain the atmospheric $\mathrm{CH}_{4} / \mathrm{CO}_{2}$ ratio to test whether the haze is present at an anomalously low ratio unexplainable by methane photochemistry alone. Although $S_{\text {org }}$ gases themselves are difficult to detect in a planet's spectrum, organic haze produces a very strong absorption feature at UV-blue wavelengths. This haze could also be detected in the NIR and mid-IR by transit transmission observations. Methane and carbon dioxide produce absorption features in the NIR that could be detected in both reflected light and transit transmission observations. Future observatories and telescope concepts such as JWST, LUVOIR, and HabEx may be able to make measurements of these spectral features. Because the haze absorption feature is so strong, it may be one of the most detectable spectral beacons of life, although interpreting the haze absorption feature in the context of its environment may be more challenging. The long anoxic history of our planet teaches us not to ignore the challenge of life detection on anoxic worlds when we design the instruments, plan the observations, and search for life on distant exoplanets.

\section{Acknowledgments}

This work was performed as part of the NASA Astrobiology Institute's Virtual Planetary Laboratory, supported by the National Aeronautics and Space Administration through the NASA Astrobiology Institute under solicitation NNH12ZDA002C and Cooperative Agreement Number NNA13AA93A. Simulations were facilitated through the use of the Hyak supercomputer system at the University of Washington eScience Institute. We thank Dr. T. McCollom for pointing us to sources discussing methane in serpentinizing systems. Spectra shown in this work will be archived at the Virtual Planetary Laboratory online spectral database. We thank our two anonymous reviewers for their helpful comments and suggestions that improved our manuscript.

\section{Author Disclosure Statement}

No competing financial interests exist.

\section{References}

Allen, M., Pinto, J.P., and Yung, Y.L. (1980) Titan: aerosol photochemistry and variations related to the sunspot cycle. Astrophys J 242:L125-L128.

Anbar, A.D., Yung, Y.L., and Chavez, F.P. (1996) Methyl bromide: ocean sources, ocean sinks, and climate sensitivity. Global Biogeochem Cycles 10:175-190. 
Arney, G., Domagal-Goldman, S.D., Meadows, V.S., Wolf, E.T., Schwieterman, E., Charnay, B., Claire, M., Hébrard, E., and Trainer, M.G. (2016) The pale orange dot: the spectrum and habitability of hazy Archean Earth. Astrobiology 16:873899.

Arney, G.N., Meadows, V.S., Domagal-Goldman, S.D., Deming, D., Robinson, T.D., Tovar, G., Wolf, E.T., and Schwieterman, E. (2017) Pale orange dots: the impact of organic haze on the habitability and detectability of Earthlike exoplanets. Astrophys J 836, doi:10.3847/1538-4357/836/1/49.

Beichman, C., Benneke, B., Knutson, H., Smith, R., Lagage, P.O., Dressing, C., Latham, D., Lunine, J., Birkmann, S., Ferruit, P., Giardino, G., Kempton, E., Carey, S., Krick, J., Deroo, P.D., Mandell, A., Ressler, M.E., Shporer, A., Swain, M., Vasisht, G., Ricker, G., Bouwman, J., Crossfield, I., Greene, T., Howell, S., Christiansen, J., Ciardi, D., Clampin, M., Greenhouse, M., Sozzetti, A., Goudfrooij, P., Hines, D., Keyes, T., Lee, J., McCullough, P., Robberto, M., Stansberry, J., Valenti, J., Rieke, M., Rieke, G., Fortney, J., Bean, J., Kreidberg, L., Ehrenreich, D., Deming, D., Albert, L., Doyon, R., and Sing, D. (2014) Observations of transiting exoplanets with the James Webb Space Telescope (JWST). Publ Astron Soc Pac 126:1134-1173.

Blake, N., Blake, D.R., Sive, B.C., Chen, T., Sherwood Rowland, F., Collins, J.E.J., Sachse, G.W., and Anderson, B.E. (1996) Biomass burning emissions and vertical distribution of atmospheric methyl halides and other reduced carbon gases in the South Atlantic region. J Geophys Res: Atmospheres 101: 24151-24164.

Bolcar, M.R., Balasubramanian, K., Clampin, M., Crooke, J., Feinberg, L., Postman, M., Quijada, M., Rauscher, B., Redding, D., Rioux, N., Shaklan, S., Stahl, H.P., Stahle, C., and Thronson, H. (2015) Technology development for the Advanced Technology Large Aperture Space Telescope (ATLAST) as a candidate Large UV-Optical-Infrared (LUVOIR) surveyor. Proc SPIE 9602, doi:10.1117/12.2188559.

Botet, R., Rannou, P., and Cabane, M. (1997) Mean-field approximation of Mie scattering by fractal aggregates of identical spheres. Appl Opt 36:8791-8797.

Bradley, A.S. and Summons, R.E. (2010) Multiple origins of methane at the Lost City hydrothermal field. Earth Planet Sci Lett 297:34-41.

Brazelton, W.J., Schrenk, M.O., Kelley, D.S., and Baross, J.A. (2006) Methane- and sulfur-metabolizing microbial communities dominate the Lost City hydrothermal field ecosystem. Appl Environ Microbiol 72:6257-6270.

Carr, M.H., Belton, M.J.S., Chapman, C.R., Davies, M.E., Geissler, P., Greenberg, R., McEwen, A.S., Tufts, B.R., Greeley, R., Sullivan, R., Head, J.W., Pappalardo, R.T., Klaasen, K.P., Johnson, T.V., Kaufman, J., Senske, D., Moore, J., Neukum, G., Schubert, G., Burns, J.A., Thomas, P., and Veverka, J. (1998) Evidence for a subsurface ocean on Europa. Nature 391:363-365.

Claire, M.W., Sheets, J., Cohen, M., Ribas, I., Meadows, V.S., and Catling, D.C. (2012) The evolution of solar flux from $0.1 \mathrm{~nm}$ to $160 \mu \mathrm{m}$ : quantative estimates for planetary studies. Astrophys $J$ 757, doi:10.1088/0004-637X/757/1/95.

Claire, M.W., Kasting, J.F., Domagal-Goldman, S.D., Stüeken, E.E., Buick, R., and Meadows, V.S. (2014) Modeling the signature of sulfur mass-independent fractionation produced in the Archean atmosphere. Geochim Cosmochim Acta 141: 365-380.

Core Writing Team, Pachauri, R.K., and Reisinger, A., editors. (2007) Climate Change 2007: Synthesis Report, Contribution of Working Groups I, II and III to the fourth assessment report of the Intergovernmental Panel on Climate Change, IPCC, Geneva, Switzerland.

Crisp, D. (1997) Absorption of sunlight by water vapor in cloudy conditions: a partial explanation for the cloud absorption anomaly. Geophys Res Lett 24:571-574.

Dalcanton, J., Seager, S., Aigrain, S., Hirata, C., Battel, S., Mather, J., Brandt, N., Postman, M., Conroy, C., Redding, D., and Feinberg, L. (2015) From Cosmic Birth to Living Earths: the Future of UVOIR Space Astronomy, Association of Universities for Research in Astronomy, Washington, DC.

Dauphas, N. and Kasting, J.F. (2011) Low $\mathrm{pCO}_{2}$ in the pore water, not in the Archean air. Nature 474, doi:10.1038/nature09960.

DeWitt, H.L., Trainer, M.G., Pavlov, A.A., Hasenkopf, C.A., Aiken, A.C., Jimenez, J.L., McKay, C.P., Toon, O.B., and Tolbert, M.A. (2009) Reduction in haze formation rate on prebiotic Earth in the presence of hydrogen. Astrobiology 9: 447-453.

Domagal-Goldman, S.D., Kasting, J.F., Johnston, D.T., and Farquhar, J. (2008) Organic haze, glaciations and multiple sulfur isotopes in the Mid-Archean era. Earth Planet Sci Lett 269:29-40.

Domagal-Goldman, S.D., Meadows, V.S., Claire, M.W., and Kasting, J.F. (2011) Using biogenic sulfur gases as remotely detectable biosignatures on anoxic planets. Astrobiology 11: 419-441.

Domagal-Goldman, S.D., Segura, A., Claire, M.W., Robinson, T.D., and Meadows, V.S. (2014) Abiotic ozone and oxygen in atmospheres similar to prebiotic Earth. Astrophys $J$ 792, doi: 10.1088/0004-637X/792/2/90.

Driese, S.G., Jirsa, M.A., Ren, M., Brantley, S.L., Sheldon, N.D., Parker, D., and Schmitz, M. (2011) Neoarchean paleoweathering of tonalite and metabasalt: implications for reconstructions of $2.69 \mathrm{Ga}$ early terrestrial ecosystems and paleoatmospheric chemistry. Precambrian Res 189:1-17.

Emmanuel, S. and Ague, J.J. (2007) Implications of present-day abiogenic methane fluxes for the early Archean atmosphere. Geophys Res Lett 34, doi:10.1029/2007GL030532.

Etiope, G. (2012) Climate science: methane uncovered. Nat Geosci 5:373-374.

Etiope, G. and Sherwood Lollar, B. (2013) Abiotic methane on Earth. Rev Geophys 51:276-299.

Etiope, G., Fridriksson, T., Italiano, F., Winiwarter, W., and Theloke, J. (2007) Natural emissions of methane from geothermal and volcanic sources in Europe. Journal of Volcanology and Geothermal Research 165:76-86.

France, K., Linsky, J.L., Tian, F., Froning, C.S., and Roberge, A. (2012) Time-resolved ultraviolet spectroscopy of the Mdwarf GJ 876 exoplanetary system. Astrophys J 750:L32.

Guzmán-Marmolejo, A., Segura, A., and Escobar-Briones, E. (2013) Abiotic production of methane in terrestrial planets. Astrobiology 13:550-559.

Haqq-Misra, J.D., Domagal-Goldman, S.D., Kasting, P.J., and Kasting, J.F. (2008) A revised, hazy methane greenhouse for the Archean Earth. Astrobiology 8:1127-1137.

Harman, C.E., Schwieterman, E.W., Schottelkotte, J.C., and Kasting, J.F. (2015) Abiotic $\mathrm{O}_{2}$ levels on planets around F, G, $\mathrm{K}$, and $\mathrm{M}$ stars: possible false positives for life? Astrophys $J$ 812, doi:10.1088/0004-637X/812/2/137.

Harper, D., Hamilton, J.T., Ducrocq, V., Kennedy, J.T., Downey, A., and Kalin, R.M. (2003) The distinctive isotopic signature of plant-derived chloromethane: possible application in constraining the atmospheric chloromethane budget. Chemosphere 52:433-436. 
Hasenkopf, C.A., Beaver, M.R., Trainer, M.G., Langley Dewitt, H., Freedman, M.A., Toon, O.B., McKay, C.P., and Tolbert, M.A. (2010) Optical properties of Titan and early Earth haze laboratory analogs in the mid-visible. Icarus 207:903-913.

Hasenkopf, C.A., Freedman, M.A., Beaver, M.R., Toon, O.B., and Tolbert, M.A. (2011) Potential climatic impact of organic haze on early Earth. Astrobiology 11:135-149.

Hicks, R.K., Day, D.A., Jimenez, J.L., and Tolbert, M.A. (2016) Follow the carbon: isotopic labeling studies of early Earth aerosol. Astrobiology 16:822-830.

Hörst, S.M. and Tolbert, M.A. (2014) The effect of carbon monoxide on planetary haze formation. Astrophys $J$ 781, doi: 10.1088/0004-637X/781/1/53.

$\mathrm{Hu}$, R., Seager, S., and Bains, W. (2013) Photochemistry in terresrial exoplanet atmospheres. II. $\mathrm{H}_{2} \mathrm{~S}$ and $\mathrm{SO}_{2}$ photochemimstry in anoxic atmospheres. Astrophys $J$ 769, doi: 10.1088/0004-637X/769/1/6.

Izon, G., Zerkle, A.L., Zhelezinskaia, I., Farquhar, J., Newton, R.J., Poulton, S.W., Eigenbrode, J.L., and Claire, M.W. (2015) Multiple oscillations in Neoarchaean atmospheric chemistry. Earth Planet Sci Lett 431:264-273.

Izon, G., Zerkle, A.L., Williford, K.H., Farquhar, J., Poulton, S.W., and Claire, M.W. (2017) Biological regulation of atmospheric chemistry en route to planetary oxygenation. Proc Natl Acad Sci USA 114:E2571-E2579.

Kanzaki, Y. and Murakami, T. (2015) Estimates of atmospheric $\mathrm{CO}_{2}$ in the Neoarchean-Paleoproterozoic from paleosols. Geochim Cosmochim Acta 159:190-219.

Kasting, J. and Ackerman, T. (1986) Climactic consequences of very high carbon dioxide levels in the Earth's early atmosphere. Science 234:1383-1385.

Kasting, J.F. (2005) Methane and climate during the Precambrian era. Precambrian Res 137:119-129.

Kasting, J.F. and Catling, D. (2003) Evolution of a habitable planet. Annu Rev Astron Astrophys 41:429-463.

Kasting, J.F., Liu, S.C., and Donahue, T.M. (1979) Oxygen levels in the prebiological atmosphere. J Geophys Res 84: 3097-3207.

Kelley, D.S., Karson, J.A., Fru, G.L., Yoerger, D.R., Shank, T.M., Butterfield, D.A., Hayes, J.M., Schrenk, M.O., Olson, E.J., Proskurowski, G., Jakuba, M., Bradley, A., Larson, B., Ludwig, K., Glickson, D., Buckman, K., Bradley, A.S., Brazelton, W.J., Roe, K., Elend, M.J., Delacour, A., Bernasconi, S.M., Lilley, M.D., Baross, J.A., Summons, R.E., and Sylva, S.P. (2005) A serpentinitehosted ecosystem: the Lost City hydrothermal field. Science 307:1428-1434.

Keppler, F., Eiden, R., Niedan, V., Pracht, J., and Scholer, H.F. (2000) Halocarbons produced by natural oxidation processes during degradation of organic matter. Nature 403:298-301.

Keppler, F., Harper, D.B., Röckmann, T., Moore, R.M., and Hamilton, J.T.G. (2005) New insight into the atmospheric chloromethane budget gained using stable carbon isotope ratios. Atmos Chem Phys Discuss 5:3899-3919.

Kerrich, R. and Polat, A. (2006) Archean greenstone-tonalite duality: thermochemical mantle convection models or plate tectonics in the early Earth global dynamics? Tectonophysics 415:141-165.

Kestler, D.P., Mayne, B.C., Ray, T.B., Goldstein, L.D., Brown, R.H., and Black, C.C. (1975) Biochemical components of the photosynthetic $\mathrm{CO}_{2}$ compensation point of higher plants. Biochem Biophys Res Commun 66:1439-1446.

Khare, B.N., Sagan, C., Arakawa, E.T., Suits, F., Callcott, T.A., and Williams, M.W. (1984) Optical constants of organic tholins produced in a simulated titanian atmosphere: from soft X-ray to microwave frequencies. Icarus 60:127-137.

Kharecha, P., Kasting, J., and Siefert, J. (2005) A coupled atmosphere-ecosystem model of the early Archean Earth. Geobiology 3:53-76.

Kivelson, M.G., Khurana, K.K., Russell, C.T., Volwerk, M., Walker, R.J., and Zimmer, C. (2000) Galileo magnetometer measurements: a stronger case for a subsurface ocean at Europa. Science 289:1340-1343.

Kopparapu, R.K., Ramirez, R., Kasting, J.F., Eymet, V., Robinson, T.D., Mahadevan, S., Terrien, R.C., DomagalGoldman, S., Meadows, V., and Deshpande, R. (2013) Habitable zones around main-sequence stars: new estimates. Astrophys J 765, doi10.1088/0004-637X/765/2/131.

Koppmann, R., Johen, F.J., Plass-Dulmer, C., and Rudolph, J. (1993) Distribution of methylchloride, dichloromethane, trichloroethene and tetrachloroethene over the North and South Atlantic. J Geophys Res: Atmospheres 98:20517-20526.

Kuiper, G. (1944) Titan: a satellite with an atmosphere. Astrophys $J$ 100:378.

Kurzweil, F., Claire, M., Thomazo, C., Peters, M., Hannington, M., and Strauss, H. (2013) Atmospheric sulfur rearrangement 2.7 billion years ago: evidence for oxygenic photosynthesis. Earth Planet Sci Lett 366:17-26.

Lomans, B.P., van der Drift, C., Pol, A., and Op den Camp, H.J. (2002) Microbial cycling of volatile organic sulfur compounds. Cell Mol Life Sci 59:575-588.

López-Puertas, M., Dinelli, B.M., Adriani, A., Funke, B., García-Comas, M., Moriconi, M.L., D’Aversa, E., Boersma, C., and Allamandola, L.J. (2013) Large abundances of polycyclic aromatic hydrocarbons in Titan's upper atmosphere. Astrophys J 770, doi:10.1088/0004-637X/770/2/132.

Lowell, P. (1906) Mars and Its Canals, The Macmillan Company, London.

Luger, R. and Barnes, R. (2015) Extreme water loss and abiotic $\mathrm{O}_{2}$ buildup on planets. Astrobiology 15:119-143.

Lupu, R., Marley, M., Lewis, N., Line, M., Traub, W., and Zahnle, K. (2016) Developing atmospheric retrieval methods for direct imaging spectroscopy of gas giants in reflected light. I. Methane abundances and basic cloud properties. Astron J 152, doi:10.3847/0004-6256/152/6/217.

Manley, S.L. and Dastoor, M.N. (1988) Methyl iodide $\left(\mathrm{CH}_{3} \mathrm{I}\right)$ production by kelp and associated microbes. Mar Biol 98: 477-482.

McCauley, S.E., Goldstein, A.H., and DePaolo, D.J. (1999) An isotopic approach for understanding the $\mathrm{CH}_{3} \mathrm{Br}$ budget of the atmosphere. Proc Natl Acad Sci USA 96:10006-10009.

McCollom, T.M. (2016) Abiotic methane formation during experimental serpentinization of olivine. Proc Natl Acad Sci USA 113:13965-13970.

McDermott, J.M., Seewald, J.S., German, C.R., and Sylva, S.P. (2015) Pathways for abiotic organic synthesis at submarine hydrothermal fields. Proc Natl Acad Sci USA 112:7668-7672.

Meadows, V. and Crisp, D. (1996) Ground-based near-infrared observations of the Venus nightside? The thermal structure and water abundance near the surface. J Geophys Res: Planets 101:4595-4622.

Mennesson, B., Gaudi, S., Seager, S., Cahoy, K., DomagalGoldman, S., Feinberg, L., Guyon, O., Kasdin, J., Marois, C., Mawet, D., Tamura, M., Mouillet, D., Prusti, T., Quirrenbach, A., Robinson, T., Rogers, L., Scowen, P., Somerville, R., Stapelfeldt, K., Stern, D., Still, M., Turnbull, M., Booth, J., Kiessling, A., Kuan, G., and Warfield, K. (2016) The Habitable Exoplanet (HabEx) imaging mission: preliminary sci- 
ence drivers and technical requirements. Proc SPIE 9904, doi:10.1117/12.2240457.

Misra, A., Meadows, V., Claire, M., and Crisp, D. (2014a) Using dimers to measure biosignatures and atmospheric pressure for terrestrial exoplanets. Astrobiology 14:67-86.

Misra, A., Meadows, V., and Crisp, D. (2014b) The effects of refraction on transit transmission spectroscopy: application to Earth-like exoplanets. Astrophys J 792, doi:10.1088/0004637X/792/1/61.

Misra, A., Krissansen-Totton, J., Koehler, M.C., and Sholes, S. (2015) Transient sulfate aerosols as a signature of exoplanet volcanism. Astrobiology 15:462-477.

Moran, J.J., House, C.H., Vrentas, J.M., and Freeman, K.H. (2008) Methyl sulfide production by a novel carbon monoxide metabolism in Methanosarcina acetivorans. Appl Environ Microbiol 74:540-542.

Nayak, M., Roxana, L., Marley, M., Fortney, J., Robinson, T., and Lewis, N. (2017) Atmospheric retrieval for direct imaging spectroscopy of gas giants in reflected light. II. Orbital phase and planetary radius. Publ Astron Soc Pac 129, doi: 10.1088/1538-3873/129/973/034401.

Pare, P.W. and Tumlinson, J.H. (1999) Update on plant-insect interactions: plant volatiles as a defense against insect herbivores. Plant Physiol 121:325-331.

Pavlov, A., Brown, L., and Kasting, J. (2001a) UV shielding of $\mathrm{NH}_{3}$ and $\mathrm{O}_{2}$ by organic hazes in the Archean atmosphere. $J$ Geophys Res 106:23267-23287.

Pavlov, A., Kasting, J., Eigenbrode, J., and Freeman, K. (2001b) Organic haze in Earth's early atmosphere: source of low- ${ }^{13} \mathrm{C}$ Late Archean kerogens? Geology 29:1003-1006.

Pilcher, C. (2003) Biosignatures of early Earths. Astrobiology 3: 471-486.

Planavsky, N.J., Reinhard, C.T., Wang, X., Thomson, D., McGoldrick, P., Rainbird, R.H.,... and Lyons, T.W. (2014) Low Mid-Proterozoic atmospheric oxygen levels and the delayed rise of animals. Science 346: 635-638.

Postman, M., Traub, W., Krist, J., Stapelfeldt, K., Brown, R., Oegerle, W., Lo, A., Clampin, M., Soummer, R., Wiseman, J., and Mountain, M. (2010) Advanced Technology LargeAperture Space Telescope (ATLAST): characterizing habitable worlds. ASP Conference Series 430:361-367.

Rannou, P., Cabane, M., Botet, R., and Chassèfiere, E. (1997) A new interpretation of scattered light measurements at Titan's limb. J Geophys Res 102:10997-11013.

Rasmussen, R.A., Khalili, M.A.K., Gunawardena, R., and Hoyt, S.D. (1982) Atmospheric methyl iodide $\left(\mathrm{CH}_{3} \mathrm{I}\right)$. J Geophys Res: Oceans 87:3086-3090.

Rhew, R. (2011) Sources and sinks of methyl bromide and methyl chloride in the tallgrass prairie: applying a stable isotope tracer technique over highly variable gross fluxes. J Geophys Res: Biogeosciences 116, doi:10.1029/ 2011JG001704.

Robinson, T.D., Meadows, V.S., and Crisp, D. (2010) Detecting oceans on extrasolar planets using the glint effect. Astrophys J 721:L67-L71.

Robinson, T.D., Meadows, V.S., Crisp, D., Deming, D., A'hearn, M.F., Charbonneau, D., Livengood, T.A., Seager, S., Barry, R.K., Hearty, T., Hewagama, T., Lisse, C.M., McFadden, L.A., and Wellnitz, D.D. (2011) Earth as an extrasolar planet: Earth model validation using EPOXI earth observations. Astrobiology 11:393-408.

Robinson, T.D., Ennico, K., Meadows, V.S., Sparks, W., Bussey, D.B.J., Schwieterman, E.W., and Breiner, J. (2014) Detection of ocean glint and ozone absorption using LCROSS
Earth observations. Astrophys J 787, doi:10.1088/0004-637X/ $787 / 2 / 171$

Robinson, T.D., Stapelfeldt, K.R., and Marley, M.S. (2016) Characterizing rocky and gaseous exoplanets with 2-meter class space-based coronagraphs. Publ Astron Soc Pac 128, doi:10.1088/1538-3873/128/960/025003.

Rosing, M.T., Bird, D.K., Sleep, N.H., and Bjerrum, C.J. (2010) No climate paradox under the faint early Sun. Nature 464: 744-747.

Rothman, L.S., Gordon, I.E., Babikov, Y., Barbe, A., Chris Benner, D., Bernath, P.F., Birk, M., Bizzocchi, L., Boudon, V., Brown, L.R., Campargue, A., Chance, K., Cohen, E.A., Coudert, L.H., Devi, V.M., Drouin, B.J., Fayt, A., Flaud, J.-M., Gamache, R.R., Harrison, J.J., Hartmann, J.-M., Hill, C., Hodges, J.T., Jacquemart, D., Jolly, A., Lamouroux, J., LeRoy, R.J., Li, G., Long, D.A., Lyulin, O.M., Mackie, C.J., Massie, S.T., Mikhailenko, S., Müller, H.S.P., Naumenko, O.V., Nikitin, A.V., Orphal, J., Perevalov, V., Perrin, A., Polovtseva, E.R., Richard, C., Smith, M.A.H., Starikova, E., Sung, K., Tashkun, S., Tennyson, J., Toon, G.C., Tyuterev, Vl.G., and Wagner, G. (2013) The HITRAN2012 molecular spectroscopic database. J Quant Spectrosc Radiat Transf 130:4-50.

Sagan, C. and Chyba, C. (1997) The early faint young Sun paradox: organic shielding of ultraviolet-labile greenhouse gases. Science 276:1217-1221.

Schwieterman, E.W., Meadows, V.S., Domagal-Goldman, S.D., Deming, D., Arney, G.N., Luger, R., Harman, C.E., Misra, A., and Barnes, R. (2016) Identifying planetary biosignature impostors: spectral features of $\mathrm{CO}$ and $\mathrm{O}_{4}$ resulting from abiotic $\mathrm{O}_{2} / \mathrm{O}_{3}$ production. Astrophys $J$ 819, doi:10.3847/ 2041-8205/819/1/L13.

Seager, S., Schrenk, M., and Bains, W. (2012) An astrophysical view of Earth-based metabolic biosignature gases. Astrobiology 12:61-82.

Segura, A., Kasting, J.F., Meadows, V., Cohen, M., Scalo, J., Crisp, D., Butler, R.A.H., and Tinetti, G. (2005) Biosignatures from Earth-like planets around M dwarfs. Astrobiology 5:706-725.

Shaw, G.H. (2008) Earth's atmosphere-Hadean to early Proterozoic. Chemie der Erde - Geochemistry 68:235-264.

Sive, B.C., Varner, R.K., Mao, H., Blake, D.R., Wingenter, O.W., and Talbot, R. (2007) A large terrestrial source of methyl iodide. Geophys Res Lett 34:6-10.

Solomon, S.J., Custer, T., Schade, G., Soares Dias, A.P., and Burrows, J. (2005) Atmospheric methanol measurement using selective catalytic methanol to formaldehyde conversion. Atmos Chem Phys Discuss 5:3533-3559.

Squyres, S.W., Reynolds, R.T., Cassen, P.M., and Peale, S.J. (1983) Liquid water and active resurfacing on Europa. Nature 301:225-226.

Stapelfeldt, K.R., Dekens, F.G., Brenner, M.P., Warfield, K.R., Belikov, R., Brugarolas, P.B., Bryden, G., Cahoy, K.L., Chakrabarti, S., Dubovitsky, S., Effinger, R.T., Hirsch, B., Kissil, A., Krist, J.E., Lang, J.J., Marley, M.S., McElwain, M.W., Meadows, V.S., Nissen, J., Oseas, J.M., Pong, C., Serabyn, E., Sunada, E., Trauger, J.T., and Unwin, S.C. (2015) Exo-C: a probe-scale space observatory for direct imaging and spectroscopy of extrasolar planetary systems. Proc SPIE 9605, doi:10.1117/12.2191720.

Stevenson, D. (2000) Europa's ocean-the case strengthens. Science 289:1305-1307.

Stoner, D.L., Burbank, N.S., and Miller, K.S. (1994) Anaerobic transformation of organosulfur compounds in microbial mats from Octopus Spring. Geomicrobiology 12:195-202. 
Tolbert, N.E., Benker, C., and Beck, E. (1995) The oxygen and carbon dioxide compensation points of $\mathrm{C} 3$ plants: possible role in regulating atmospheric oxygen. Proc Natl Acad Sci USA 92:11230-11233.

Trainer, M.G., Pavlov, A.A., DeWitt, H.L., Jimenez, J.L., McKay, C.P., Toon, O.B., and Tolbert, M.A. (2006) Organic haze on Titan and the early Earth. Proc Natl Acad Sci USA 103:18035-18042.

Turnbull, M.C., Glassman, T., Roberge, A., Cash, W., Noecker, C., Lo, A., Mason, B., Oakley, P., and Bally, J. (2012) The search for habitable worlds. 1. The viability of a starshade mission. Publ Astron Soc Pac 124, doi:10.1086/666325.

Ueno, Y., Yamada, K., Yoshida, N., Maruyama, S., and Isozaki, Y. (2006) Evidence from fluid inclusions for microbial methanogenesis in the early Archaean era. Nature 440:516-519.

Vance, S., Christensen, L.E., Webster, C.R., and Sung, K. (2011) Volatile organic sulfur compounds as biomarkers complementary to methane: infrared absorption spectroscopy of $\mathrm{CH}_{3} \mathrm{SH}$ enables in situ measurements on Earth and Mars. Planet Space Sci 59:299-303.

Ver Eecke, H.C., Butterfield, D.A., Huber, J.A., Lilley, M.D., Olson, E.J., Roe, K.K., Evans, L.J., Merkel, A.Y., Cantin, H.V., and Holden, J.F. (2012) Hydrogen-limited growth of hyperthermophilic methanogens at deep-sea hydrothermal vents. Proc Natl Acad Sci USA 109:13674-13679.

Von Braun, K., Boyajian, T.S., van Belle, G.T., Kane, S.R., Jones, J., Farrington, C., Schaefer, G., Vargas, N., Scott, N., ten Brummelaar, T.A., Kephart, M., Gies, D.R., Ciardi, D.R., Lopez-Morales, M., Mazingue, C., McAlister, H.A., Ridgway, S., Goldfinger, P.J., Turner, N.H., and Sturmann, L. (2014) Stellar diameters and temperatures-V. 11 newly characterized exoplanet host stars. Mon Not $R$ Astron Soc 438:2413-2425.

Vuitton, V., Tran, B.N., Persans, P.D., and Ferris, J.P. (2009) Determination of the complex refractive indices of Titan haze analogs using photothermal deflection spectroscopy. Icarus 203:663-671.

Waite, J.H., Young, D.T., Cravens, T.E., Coates, A.J., Crary, F.J., Magee, B., and Westlake, J. (2007) The process of tholin formation in Titan's upper atmosphere. Science 316: 870-875.

Walser, M.L., Park, J., Gomez, A.L., Russell, A.R., and Nizkorodov, S.A. (2007) Photochemical aging of secondary organic aerosol particles generated from the oxidation of dlimonene. J Phys Chem A 111:1907-1913.

Went, F.W. (1960) Blue hazes in the atmosphere. Nature 187: 641-643.
Woese, C.R. and Fox, G.E. (1977) Phylogenetic structure of the prokaryotic domain: the primary kingdoms. Proc Natl Acad Sci USA 74:5088-5090.

Wolf, E.T. and Toon, O.B. (2010) Fractal organic hazes provided an ultraviolet shield for early Earth. Science 328:12661268.

Xin, J.-Y., Cui, J., Niu, J., Hua, S., Xia, C., and Li, S. (2004) Production of methanol from methane by methanotrophic bacteria. Biocatal Biotransformation 22:225-229.

Yokouchi, Y., Noijiri, Y., Barrie, L.A., Toom-Sauntry, D., Machida, T., Inuzuka, Y., Akimoto, H., Li, H.J., Fujinuma, Y., and Aoki, S. (2000) A strong source of methyl chloride to the atmosphere from tropical coastal land. Nature 403:295-298.

Yung, Y.L., Allen, M., and Pinto, J.P. (1984) Photochemistry of the atmosphere of Titan: comparison between model and observations. Astrophys J Suppl Ser 55:465-506.

Zahnle, K., Claire, M., and Catling, D. (2006) The loss of massindependent fractionation in sulfur due to a Palaeoproterozoic collapse of atmospheric methane. Geobiology 4:271-283.

Zerkle, A.L., Claire, M.W., Domagal-Goldman, S.D., Farquhar, J., and Poulton, S.W. (2012) A bistable organic-rich atmosphere on the Neoarchaean Earth. Nat Geosci 5:359-363.

Address correspondence to: Giada Arney NASA Goddard Space Flight Center Mail Code 693 8800 Greenbelt Road Greenbelt, MD 20771

E-mail: giada.n.arney@nasa.gov

Submitted 8 March 2017 Accepted 20 September 2017

\begin{tabular}{rl|} 
Abbreviations Used \\
HabEx $=$ Habitable Exoplanet Imaging Mission \\
IWA $=$ inner working angle \\
JWST $=$ James Webb Space Telescope \\
LUVOIR $=$ Large UV Optical Infrared telescope \\
NIR $=$ near infrared \\
SMART $=$ Spectral Mapping Atmospheric \\
Radiative Transfer model \\
SNR $=$ signal-to-noise ratio
\end{tabular}

